\title{
Structural basis for the neutralization of SARS-CoV-2 by an antibody from a convalescent patient
}

\author{
Daming Zhou 1,17, Helen M. E. Duyvesteynn,17, Cheng-Pin Chen ${ }^{2,17}$, Chung-Guei Huang, ${ }^{3,4}$, Ting-Hua Chen ${ }^{5}$, \\ Shin-Ru Shih ${ }^{3,4}$, Yi-Chun Lin ${ }^{6}$, Chien-Yu Cheng, ${ }^{2}$ Shu-Hsing Cheng ${ }^{6}$, Yhu-Chering Huang7,

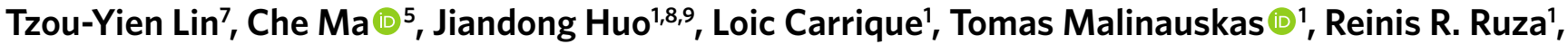 \\ Pranav N. M. Shah', Tiong Kit Tan ${ }^{10}{ }^{10}$, Pramila Rijal ${ }^{10,11}$, Robert F. Donat ${ }^{10}$, Kerry Godwin ${ }^{12}$, \\ Karen R. Buttigieg ${ }^{12}$, Julia A. Tree ${ }^{12}$, Julika Radecke ${ }^{13}$, Neil G. Paterson ${ }^{13}{ }^{13}$, Piyada Supasa ${ }^{14}$, \\ Juthathip Mongkolsapaya ${ }^{14,15}$, Gavin R. Screaton ${ }^{14}$, Miles W. Carroll'2,14, Javier Gilbert-Jaramillo ${ }^{16}$, \\ Michael L. Knight $\mathbb{1}{ }^{16}$, William James ${ }^{16}{ }^{16}$, Raymond J. Owens $\mathbb{1}{ }^{1,8,9}$, James H. Naismith $\mathbb{D}^{1,8,9}$, \\ Alain R. Townsend ${ }^{10,11}$, Elizabeth E. Fry ${ }^{(1)}$, Yuguang Zhao ${ }^{1,17}$, Jingshan Ren ${ }^{1}$, , David I. Stuart $\mathbb{1}^{1,11,13 凶}$ \\ and Kuan-Ying A. Huang $\mathbb{B}^{3,7} \bowtie$
}

The COVID-19 pandemic has had an unprecedented health and economic impact and there are currently no approved therapies. We have isolated an antibody, EY6A, from an individual convalescing from COVID-19 and have shown that it neutralizes SARS-CoV-2 and cross-reacts with SARS-CoV-1. EY6A Fab binds the receptor binding domain (RBD) of the viral spike glycoprotein tightly $\left(K_{\mathrm{D}}\right.$ of $\left.2 \mathrm{nM}\right)$, and a 2.6-A-resolution crystal structure of an RBD-EY6A Fab complex identifies the highly conserved epitope, away from the ACE2 receptor binding site. Residues within this footprint are key to stabilizing the pre-fusion spike. Cryo-EM analyses of the pre-fusion spike incubated with EY6A Fab reveal a complex of the intact spike trimer with three Fabs bound and two further multimeric forms comprising the destabilized spike attached to Fab. EY6A binds what is probably a major neutralizing epitope, making it a candidate therapeutic for COVID-19.

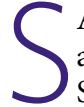
ARS-CoV-2 was first detected in December 2019, leading to a pandemic with an estimated $5-6 \%$ mortality rate ${ }^{1}$. Akin to SARS-CoV-1, the causative agent of the 2003 SARS outbreak, this is an enveloped betacoronavirus with protrusions of large trimeric 'spike' proteins. Receptor binding domains (RBDs) located at the tips of these spikes facilitate host cell entry via interaction with angiotensin-converting enzyme $2(\mathrm{ACE} 2)^{2}$. Spikes are type I transmembrane glycoproteins, formed from a single polypeptide, which transitions into a post-fusion state via cleavage into S1 (N-terminal) and S2 (C-terminal) chains following receptor binding or trypsin treatment ${ }^{3}$. In the pre-fusion state, the apical RBD $(\sim 22 \mathrm{kDa})$ is folded down, enshrouded by the N-terminal domain (NTD) of the spike so that the receptor binding site is inaccessible until, it is assumed, an RBD stochastically swings upwards to
\end{abstract}

present the ACE2 binding site ${ }^{4-7}$. ACE2 interaction locks the RBD in the 'up' conformation, which drives conversion to the post-fusion form where the S2 subunit engages the host membrane while dispensing with $S 1^{4,5}$.

Neutralizing human monoclonal antibodies (mAbs) that recognize the ACE2 receptor binding site for SARS-CoV-1 and SARS-CoV-2 are generally not cross-reactive between the two viruses and are susceptible to escape mutation ${ }^{8-12}$. Indeed, a natural mutation (Y495N) has already been identified at this site (GISAID $^{13}$ : accession ID: EPI_ISL_429783 Wienecke-Baldacchino et al.). By contrast, the CR3022 antibody (derived from a SARS-CoV1-infected patient) cross-reacts strongly with SARS-CoV-2 (see Methods and Fig. 1) and has been shown to recognize a cryptic, conserved footprint on the RBD distinct from the binding epitope of

'Division of Structural Biology, Nuffield Department of Medicine, University of Oxford, The Wellcome Centre for Human Genetics, Headington, Oxford, UK. ${ }^{2}$ Department of Infectious Diseases, Taoyuan General Hospital, Ministry of Health and Welfare, Taoyuan, and National Yang-Ming University, Taipei, Taiwan. ${ }^{3}$ Research Center for Emerging Viral Infections, College of Medicine, Chang Gung University, Taoyuan, Taiwan. ${ }^{4}$ Department of Laboratory Medicine, Chang Gung Memorial Hospital, Taoyuan, Taiwan. ${ }^{5}$ Genomics Research Center, Academia Sinica, Taipei, Taiwan. ${ }^{6}$ Department of Infectious Diseases, Taoyuan General Hospital, Ministry of Health and Welfare, Taoyuan, and Taipei Medical University, Taipei, Taiwan. ${ }^{7}$ Division of Pediatric Infectious Diseases, Department of Pediatrics, Chang Gung Memorial Hospital, Taoyuan, Taiwan. ${ }^{8}$ The Rosalind Franklin Institute, Harwell Campus, Didcot, UK. ${ }^{9}$ Protein Production UK, Research Complex at Harwell, Harwell Science \& Innovation Campus, Didcot, UK. ${ }^{10} \mathrm{MRC}$ Human Immunology Unit, Weatherall Institute of Molecular Medicine, University of Oxford, John Radcliffe Hospital, Oxford, UK. "Centre for Translational Immunology, Chinese Academy of Medical Sciences Oxford Institute, University of Oxford, Oxford, UK. ${ }^{2}$ National Infection Service, Public Health England, Porton Down, Salisbury, UK. ${ }^{13}$ Diamond Light Source Ltd, Harwell Science \& Innovation Campus, Didcot, UK. ${ }^{14}$ Nuffield Department of Medicine, Wellcome Centre for Human Genetics, University of Oxford, Oxford, UK. ${ }^{15}$ Dengue Hemorrhagic Fever Research Unit, Office for Research and Development, Faculty of Medicine, Siriraj Hospital, Mahidol University, Bangkok, Thailand. ${ }^{16}$ William Dunn School of Pathology, University of Oxford, Oxford, UK. ${ }^{17}$ These authors contributed

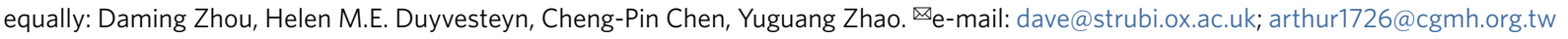



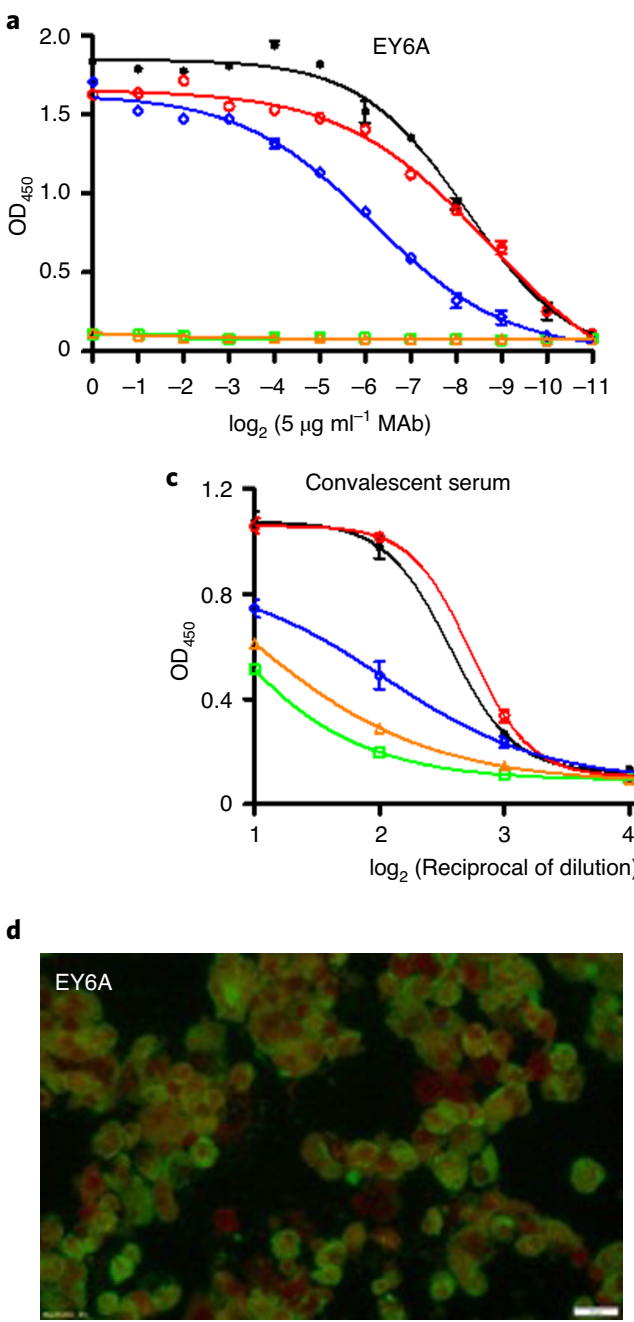

d

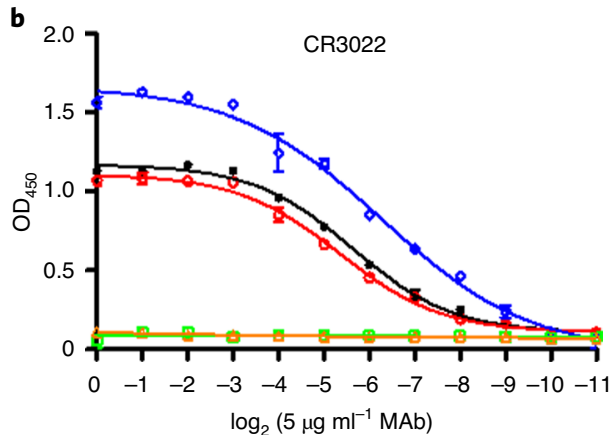

$$
\begin{aligned}
& \rightarrow \text { SARS-CoV-2 spike } \\
& \rightarrow \text { SARS-CoV-2 S1 } \\
& \rightarrow \text { SARS-CoV-1 S1 } \\
& \because \text { MERS spike } \\
& \leftarrow \text { OC43 spike }
\end{aligned}
$$

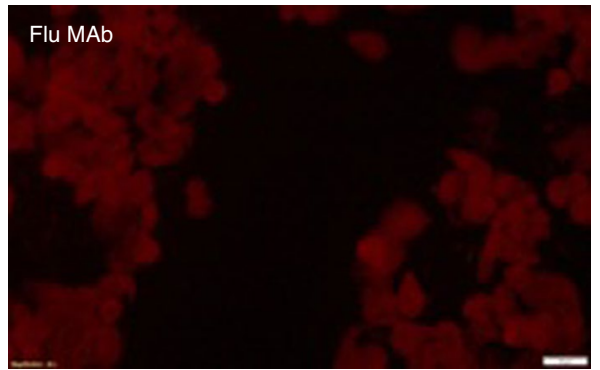

Fig. 1 | Binding specificity of EY6A in ELISA and immunofluorescence. a, ELISA curves showing that antibody EY6A binds the S1 subunit of SARS-CoV-2 and cross-reacts with S1 of SARS-CoV-1. b. Similarly, antibody CR3022 binds the S1 subunit of SARS-CoV-1 and cross-reacts with SARS-CoV-2 S1, but with lower affinity. c, Convalescent serum from a patient with COVID-19 used as a control showed binding to SARS-CoV-1, SARS-CoV-2, MERS and OC43 spike proteins. d, Indirect immunofluorescence assay. Antibody EY6A bound to viral antigens expressed on SARS-CoV-2-infected cells, as visualized by apple-green fluorescence against a background of red fluorescing material stained by the Evans Blue counterstain (left). Anti-influenza H3 MAb BS 1A was included as a control (right). Images were acquired with an original magnification of $\times 40 . \mathrm{OD}_{450}$, optical density at $450 \mathrm{~nm}$. Scale bars, $20 \mu \mathrm{m}$.

ACE2 $2^{9,14-16}$. That this is not uncommon for SARS-CoV-1 antibodies is suggested by similar observations for the $47 \mathrm{D} 11$ antibody ${ }^{17}$. We set out to characterize the antibody response in individuals infected with SARS-CoV-2. One antibody, EY6A, identified from a convalescent patient was found to recognize the RBD and shown to be highly neutralizing. We determined the structure of this antibody Fab in complex with the viral antigen to ascertain its mechanism of action.

\section{Results}

EY6A binds SARS-CoV-2 spike and S1. To isolate SARS-CoV-2 spike-reactive $\mathrm{mAbs}$, we cloned antibody genes from blood-derived plasmablasts of a patient with COVID-19 in the convalescent phase. This 43-year-old male patient was hospitalized with febrile illness and developed pneumonia during admission. The pneumonia resolved within days without requiring respiratory support and the patient recovered fully. The plasmablasts were isolated two weeks after onset of illness for mAb cloning. One of those mAbs, EY6A, was shown by ELISA to bind full-length spike or purified S1 protein of SARS-CoV-2 and to cross-react with SARS-CoV-1 (although with lower affinity) (Fig. 1a). This is analogous to antibody CR3022 $2^{14,15}$, isolated from a patient with SARS-CoV-1, which shows a lower apparent affinity for SARS-CoV-2 spike or S1 than EY6A, but higher against SARS-CoV-1 S1 (Fig. 1b). Although cross-reactive with SARS-CoV-1, neither EY6A nor CR3022 recognize spike from other coronaviruses (MERS and OC43) (Fig. 1a,b), but convalescent sera (used as a control, Fig. 1c) can recognize spike from MERS and OC43. Binding of EY6A to viral antigens expressed on SARS-CoV-2-infected Vero E6 cells was detected by immunofluorescence (Fig. 1d).

EY6A binds to spike RBD at a site spatially separated from that of ACE2. Surface plasmon resonance (SPR) measurements showed high-affinity binding of EY6A Fab to immobilized SARS-CoV-2 $\mathrm{RBD}$ (as an RBD-Fc construct, $K_{\mathrm{D}}=2 \mathrm{nM}$, Extended Data Fig. 1a), whereas the value derived from the kinetic data for immobilized EY6A immunoglobulin-G (IgG) was somewhat higher (Extended Data Fig. $1 \mathrm{~b}$ and Supplementary Table 1). SPR analysis also revealed competition between EY6A and CR3022 binding to RBD, such that incubation of RBD with either antibody eliminated binding to the other, immobilized on the chip (Extended Data Fig. 2a,c). The competition between CR3022 and EY6A for RBD binding suggests that these two antibodies have the same or overlapping footprints. 

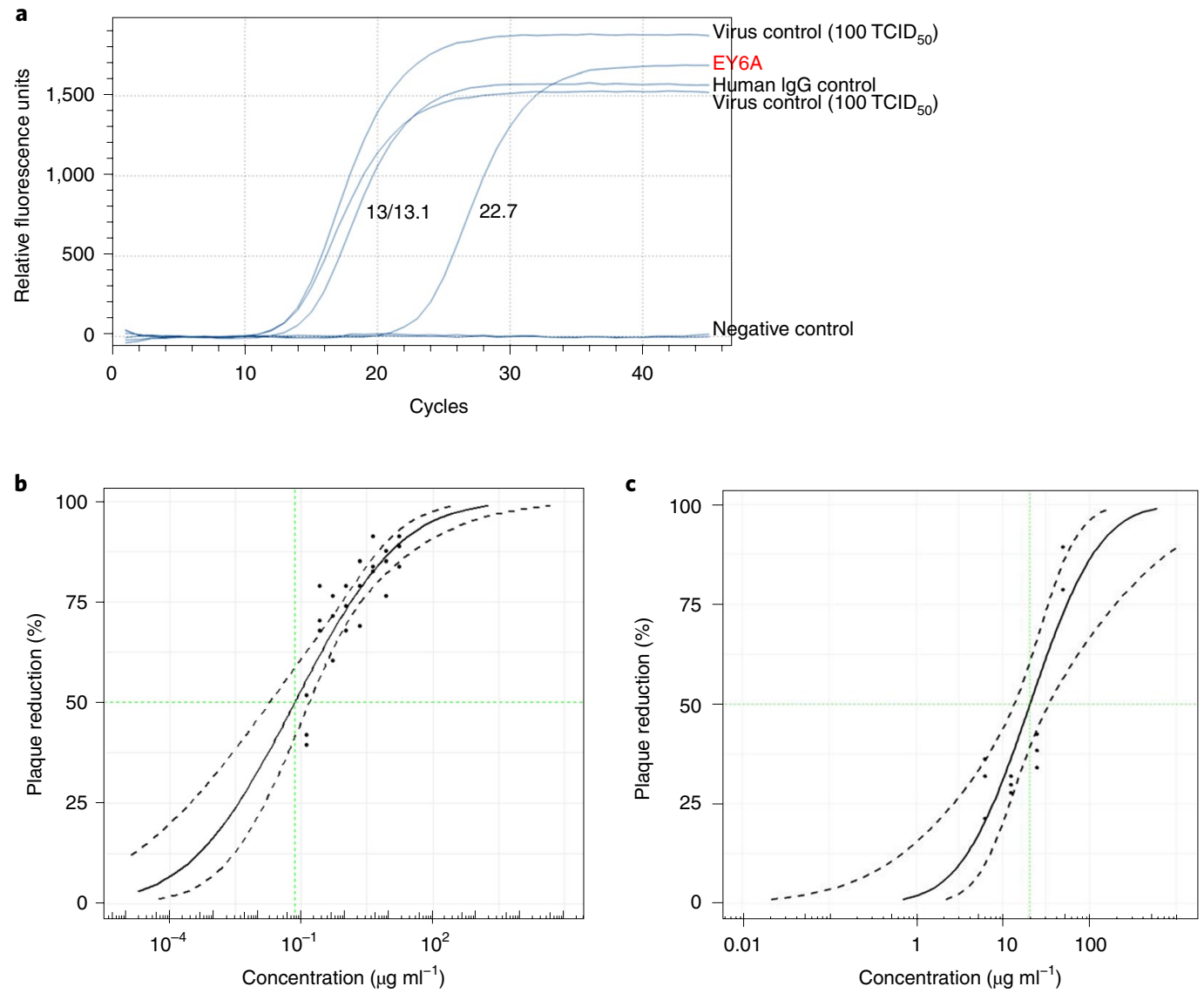

Fig. 2 | Neutralization of SARS-CoV-2 by EY6A. a, Neutralization data acquired by measuring the Ct (threshold cycle) value of virus signal in the supernatant of SARS-CoV-2-infected Vero E6 cells in an E gene-based real-time PCR assay with reverse transcription ${ }^{28}$. An increase indicates a decrease in virus template. Each unit increase suggests a $2 \times$ reduction resulting from the presence of Mab. An $\sim 10 \times$ increase in Ct corresponds to an $~ 1,000$-fold reduction of viral nucleic acid copies. Virus control was at 100 TCID $_{50}$ (median tissue culture infectious dose). Anti-influenza H3 MAb BS $1 \mathrm{~A}$ was included as a human IgG control in the assay, and both this and EY6A were used at $1.5 \mu \mathrm{g} \mathrm{ml}^{-1}$. The $\mathrm{Ct}$ values are marked in the plot. The neutralization assay was carried out twice with equivalent results. b. Dose-response curve for PRNT with EY6A at a starting concentration of $2.7 \mathrm{mg} \mathrm{ml}^{-1}$. The probit mid-point is $0.071 \mu \mathrm{ml}^{-1}$ (confidence intervals: $0.019-0.151 \mu \mathrm{g} \mathrm{ml}^{-1}$ ). The Excel Spearman-Kärber ND 50 ( $50 \%$ neutralizing dose) is $0.39 \mu \mathrm{g} \mathrm{ml}{ }^{-1}$. c, Vero-cell-based PRNT assay showing neutralization of SARS-CoV-2 by EY6A and CR3022. The probit mid-point is $20.7 \mu \mathrm{g} \mathrm{ml}^{-1}$ (confidence intervals: $13.7-34.1 \mu \mathrm{g} \mathrm{ml}{ }^{-1}$ ). The Excel Spearman-Kärber $\mathrm{ND}_{50}$ is $10 \mu \mathrm{g} \mathrm{ml}^{-1}$. Three technical replicates were done and the confidence limits were calculated as defined in ref. ${ }^{18}$.

CR3022 binds to the RBD at a location distant from the ACE2 binding site, and shows a similar partial effect on ACE2 binding to $\mathrm{RBD}^{14}$. Attachment of preincubated RBD and EY6A to immobilized ACE2 indicated that, although there was substantial attachment, the off-rate from ACE2 was increased by the presence of EY6A, whereas when EY6A was immobilized on the SPR chip, preincubation of RBD with ACE2 significantly reduced binding to EY6A (Extended Data Fig. 2b,c).

To investigate this apparent partial interference between EY6A and ACE2 in a cellular context, we produced MDCK-SIAT1 cells that had either RBD or ACE2 stably expressed on their surface (MDCK-RBD or MDCK-ACE2; see Methods). We first measured the ability of EY6A to inhibit binding of the RBD to MDCK-ACE2, and found a half-maximum inhibitory concentration $\left(\mathrm{IC}_{50}\right)$ of $54 \mathrm{nM}$, which is around seven times stronger than that of Fab CR3022 $2^{15}$ $\left(\mathrm{IC}_{50}=347 \mathrm{nM}\right)$ and equivalent to soluble ACE2-Fc (Extended Data Fig. 2d). We then performed the converse analyses, and found that EY6A did not block binding of soluble, biotin-labeled ACE2 to MDCK-RBD (Extended Data Fig. 2e). These observations are consistent with an allosteric or weak direct interaction between EY6A and ACE2 as they attach to the RBD.

The reason for crosstalk between the binding of ACE2 and EY6A is not immediately obvious, because the proteins bind separate regions of the RBD and there are no steric clashes when both are docked in place on the RBD (Extended Data Fig. 3a). However, we noted that ACE2 bears two glycosylated residues (N322 and N546), which face EY6A (Extended Data Fig. 3a) and CR3022 (Extended Data Fig. 3b) in the docked models, such that bulky complex sugars on ACE2 might clash with bound antibody. We therefore repeated the SPR experiment with EY6A immobilized, and RBD and de-glycosylated ACE2 were then flowed past (Extended Data Fig. 2c). The signal was greatly enhanced following de-glycosylation, supporting the hypothesis that glycosylation of ACE2 accounts for at least part of the observed crosstalk between ACE2 and EY6A.

EY6A neutralizes SARS-CoV-2. Three distinct neutralization assays using live wild-type SARS-CoV-2 showed strong neutralization by EY6A. First, a neutralization test based on quantitative polymerase chain reaction ( $\mathrm{qPCR}$ ) detection of virus in the supernatant of infected Vero E6 cells after five days of culture showed a $\sim 1,000$-fold reduction in viral nucleic acid copies by EY6A (Fig. 2a), indicating that this antibody is highly neutralizing.

These findings were corroborated by a plaque reduction neutralization test (PRNT) at PHE Porton Down, in which EY6A showed an $\mathrm{ND}_{50}$ of $\sim 70 \mathrm{ng} \mathrm{ml}^{-1}(0.5 \mathrm{nM})$ (calculated according to ref. ${ }^{18}$; Fig. 2b, Supplementary Fig. 2 and Supplementary Table 2). 


\begin{tabular}{|c|c|c|}
\hline & $\begin{array}{l}\text { RBD-EY6A } \\
\text { (PDB 6ZER) }\end{array}$ & $\begin{array}{l}\text { RBD-EY6A-Nb } \\
\text { (PDB 6ZCZ) }\end{array}$ \\
\hline \multicolumn{3}{|l|}{ Data collection ${ }^{\mathrm{a}}$} \\
\hline Space group & P3121 & R3 \\
\hline \multicolumn{3}{|l|}{ Cell dimensions } \\
\hline$a, b, c(\AA)$ & $166.6,166.6,270.8$ & $178.1,178.1,87.8$ \\
\hline$\alpha, \beta, \gamma\left(^{\circ}\right)$ & $90,90,120$ & $90,90,120$ \\
\hline Resolution $(\AA)$ & $144-3.80(3.87-3.80)^{b}$ & $89-2.64(2.69-2.64)$ \\
\hline$R_{\text {merge }}$ & 0.227 & 0.209 \\
\hline$R_{\text {pim }}$ & $0.052(0.636)$ & $0.071(1.369)$ \\
\hline$I / \sigma(I)$ & $7.3(0.4)$ & $5.0(0.20)$ \\
\hline $\mathrm{CC}_{1 / 2}$ & $0.998(0.783)$ & $0.993(0.298)$ \\
\hline Completeness (\%) & $100(100)$ & $99.2(93.0)$ \\
\hline Redundancy & $19.8(19.8)$ & $9.4(5.3)$ \\
\hline \multicolumn{3}{|l|}{ Refinement } \\
\hline Resolution $(\AA)$ & $28.52-3.80$ & $35.3-2.65$ \\
\hline No. reflections & $40,960 / 2,156$ & $25,517 / 1,267$ \\
\hline$R_{\text {work }} / R_{\text {free }}$ & $0.212 / 0.251$ & $0.215 / 0.260$ \\
\hline \multicolumn{3}{|l|}{ No. atoms } \\
\hline Protein & 14,585 & 5,819 \\
\hline $\mathrm{NAG} / \mathrm{Cl} / \mathrm{PO}_{4}$ & 62 & 20 \\
\hline \multicolumn{3}{|l|}{$B$ factors } \\
\hline Protein & 185 & 82 \\
\hline $\mathrm{NAG} / \mathrm{Cl} / \mathrm{PO}_{4}$ & 246 & 128 \\
\hline \multicolumn{3}{|l|}{ R.m.s. deviations } \\
\hline Bond lengths $(\AA)$ & 0.004 & 0.002 \\
\hline Bond angles $\left(^{\circ}\right)$ & 0.7 & 0.4 \\
\hline
\end{tabular}

ane crystal was used for each structure. 'Values in parentheses are for the highest-resolution shell.

A separate PRNT implementation at Oxford gave a higher $\mathrm{ND}_{50}$ of $\sim 20 \mu \mathrm{g} \mathrm{ml}^{-1}$ (Fig. 2c). In both cases there was some breakthrough of the virus, so that $100 \%$ neutralization was not achieved and the difference between the two PRNT tests was more marked than would be expected from the differences in temperature and antibody incubation time (see Methods).

In summary, all tests showed neutralization and, where this was quantified, the $\mathrm{ND}_{50}$ was at weakest $20 \mu \mathrm{g} \mathrm{ml}^{-1}$, with other results indicating that it might be much stronger.

Structures of RBD-EY6A Fab complexes reveal a conserved footprint. To elucidate the footprint of EY6A, we determined the crystal structures of SARS-CoV-2 RBD (de-glycosylated to remove conformational heterogeneity) in complex with EY6A Fab. We also obtained a ternary complex with SARS-CoV-2 RBD, EY6A Fab and nanobody $(\mathrm{Nb}) \mathrm{H} 11-\mathrm{H} 4$, which has been shown to compete with $\mathrm{ACE} 2^{19}$ as a crystallization chaperone. The crystals of the binary complex diffracted to a resolution of $3.8 \AA$ and those of the ternary complex to $2.6 \AA$ (Table 1 ).

The interaction between EY6A and the RBD was identical in both complexes (Fig. 3a,b and Extended Data Fig. 4a-c). The higher-resolution ternary complex, which showed no interaction between EY6A and the Nb (Extended Data Fig. 4c and Fig. 3c), permitted a full interpretation of the detailed interactions (Extended Data Fig. 4d,e and Figs. 3 f and $4 a-d)$. Residues 333-527 of the RBD,
$1-136$ and $141-224$ of the heavy chain and 1-215 of the light chain of EY6A and 2-126 of the $\mathrm{Nb}$ are well defined and the representative electron density is shown (Extended Data Fig. 4d,e).

$\mathrm{Nb} \mathrm{H} 11-\mathrm{H} 4$ has a footprint adjacent to and slightly overlapping the ACE2 receptor binding site, and it binds the RBD orthogonally to EY6A (Fig. 3c) ${ }^{19}$. EY6A has essentially the same footprint as CR3022 $2^{14,15}$ but with a different pose, corresponding to a $73^{\circ}$ rotation around an axis perpendicular to the RBD $\alpha 3$-helix (central to both footprints) (Fig. 3d,e). The Fab complex interface buries 564 and $361 \AA^{2}$ of surface area for the complementarity determining regions (CDRs) of the heavy and light chains, respectively. The interaction is mediated by EY6A CDR loops H1, H2, H3, L1 and L3, which predominantly contact $\alpha 3$, but also $\alpha 2$ and the $\beta 2-\alpha 3$, $\alpha 4-\alpha 5$ and $\alpha 5-\beta 4$ loops of the RBD (Fig. $4 \mathrm{a}, \mathrm{b}$ and Extended Data Fig. $5 \mathrm{a}-\mathrm{g}$ ). A total of 16 residues from the heavy chain and 11 from the light chain participate in the interface, together with 31 residues from the RBD. Those heavy chain residues potentially form six hydrogen bonds and a single salt bridge between D99 (of H3) and K386 of the RBD; the light chain residues contribute an additional six hydrogen bonds. Hydrophobic interactions further increase the binding affinity (Fig. $4 \mathrm{c}, \mathrm{d})$. Of the 31 residues on RBD involved in the interaction, 21 are conserved between the CR3022 and EY6A footprints (Extended Data Fig. 5a). Overall, the area of the interaction surface and number of interactions is similar for CR3022 and EY6A and the reason for the tighter binding of EY6A is not readily apparent.

Binding of EY6A induces conformational changes into the RBD at the $\alpha 2$-helix (residues 365-371) and the $\alpha 3$-helix (residues 384-388) (Extended Data Fig. 5f,g), similar to those seen for the CR3022 complex $^{14}$. Comparison of the footprint residues for EY6A, CR3022 ${ }^{14}$ and $\mathrm{Nb}$ VHH72 $2^{20}$, shows a substantial overlap (Extended Data Fig. 5f-h), although the bulk of the molecules extend in different directions, such that VHH72 directly blocks ACE2 binding ${ }^{20}$.

The EY6A footprint forms a protein-protein interface in the pre-fusion spike. The first structures determined for the pre-fusion spike ${ }^{4,6}$ had residues 986 and 987 (in the linker between two helices in S2) mutated to a Pro-Pro sequence, to prevent the conversion to the post-fusion helical conformation. In those structures, the RBDs within the spike trimer were found in either a one 'up' two 'down' $\left(\mathrm{PDB}_{6 \mathrm{VSB}^{4}}\right.$ and $\mathrm{PDB} 6 \mathrm{VYB}^{6}$ ) or all three 'down' configuration $\left(\mathrm{PDB} 6 \mathrm{VXX}^{6}\right)$, and in both cases the EY6A epitope is inaccessible.

In the 'down' position, the footprint region is packed against another RBD of the trimer and the NTD of the neighboring protomer. More specifically, the EY6A epitope packs tightly against the S2 'knuckle' bearing the Pro-Pro mutations, forming a buried protein-protein interface and making the epitope completely inaccessible.

A preprint with structures for the wild-type spike describes a more closed form ${ }^{21}$ in which the $\mathrm{S} 1$ portion of the spike interacts more closely around the trimer axis, but that structure is not yet publicly available. We assume that, in this closer-packed form, the interprotomer interaction involving the EY6A footprint will be even tighter and most probably responsible for maintaining the spike in the pre-fusion state. Even when the RBD is in the 'up' configuration, the epitope remains largely inaccessible, and a substantial further movement of the RBD would be required to permit interaction, unless more than one RBD within the trimer is in the 'up' conformation ${ }^{14}$.

Cryo-electron microscopy shows three EY6A Fabs can insert into the spike trimer. To investigate how the EY6A interacts with spike, we performed cryo-EM analysis. Spike ectodomain was mixed with a sixfold molar excess of EY6A Fab and incubated at room temperature $\left(21^{\circ} \mathrm{C}\right)$ with an aliquot taken at $5 \mathrm{~h}$, applied to cryo-EM grids 


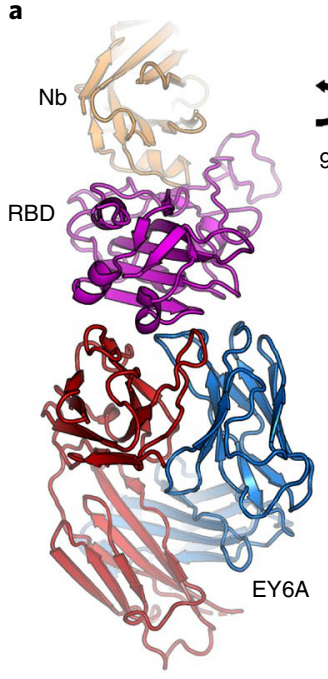

d

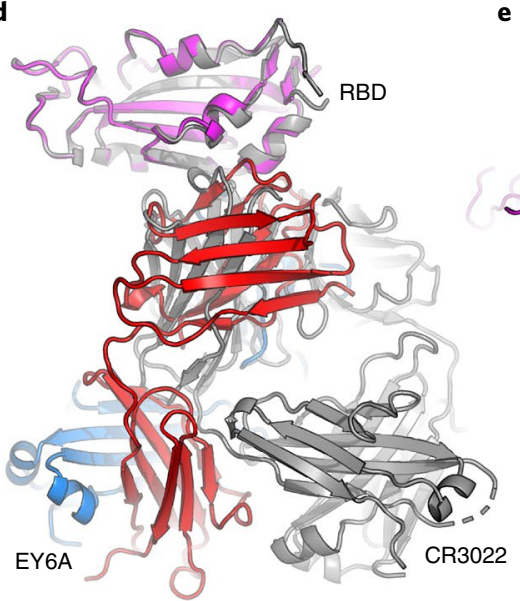

b
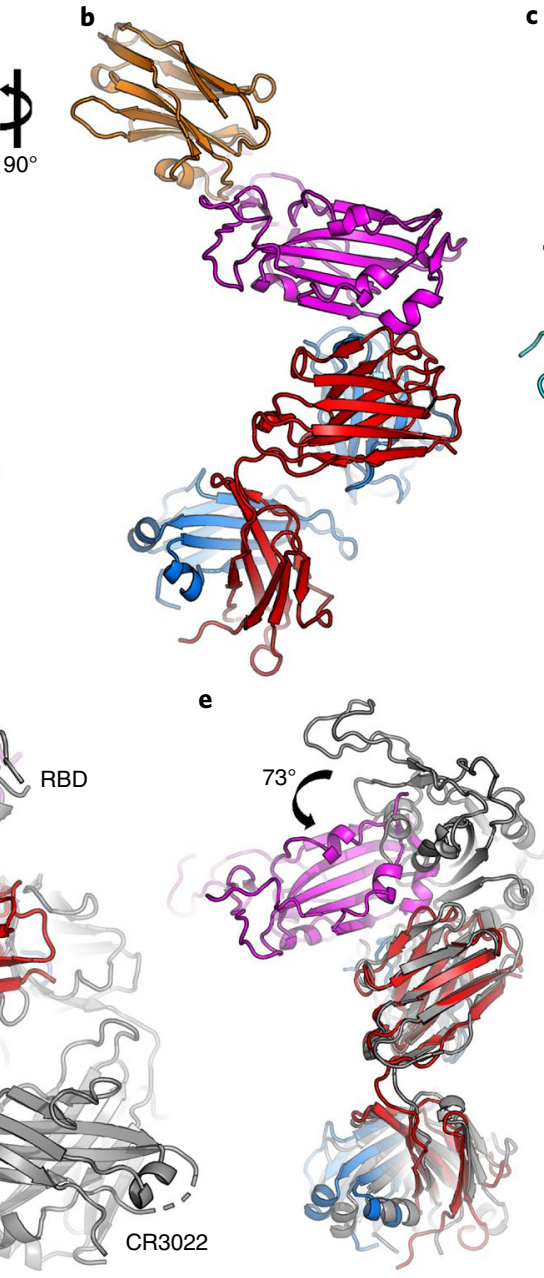

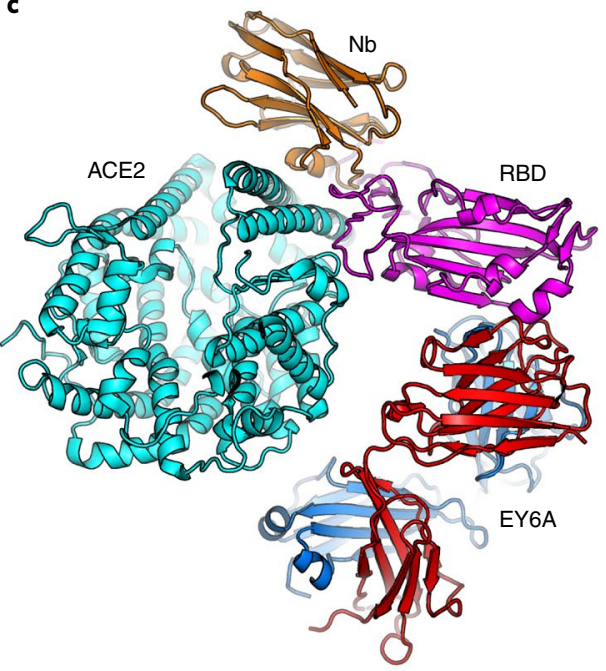

f

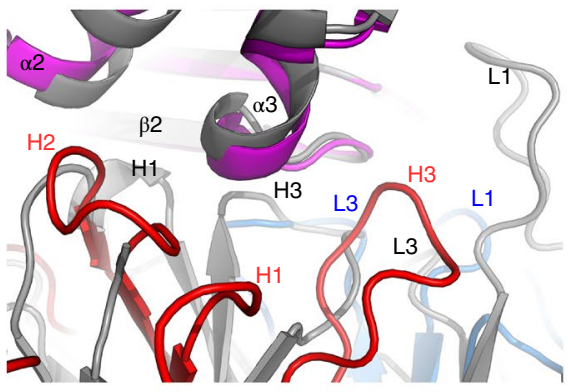

Fig. 3 | Overall structure of the RBD-EY6A complex. a, A ribbon diagram of the crystal structure of the RBD-EY6A-Nb ternary complex. The RBD, EY6A heavy and light chains and $\mathrm{Nb}$ are colored magenta, red, blue and orange, respectively. $\mathbf{b}, \mathrm{A} 90^{\circ}$ rotation of $\mathbf{a}$. c, $\mathrm{ACE} 2$ (cyan) modeled into the ternary structure by superposing the RBD of the RBD-ACE2 complex (PDB 6MOJ29) onto the ternary complex RBD. d, The RBD of the RBD-CR3022 complex (gray; PDB 6YLA ${ }^{14}$ ) superposed on the RBD of the ternary complex. e, As in $\mathbf{d}$ but superposing the Vh domain. The Nb is omitted. $\mathbf{f}$, Close-up of RBDantibody interface of $\mathbf{d}$ showing the different epitope engagements by EY6A and CR3022.

and frozen (see Methods). Unbiased two-dimensional (2D) class averages revealed three major particle classes with over one-third of the particles forming a trimeric spike-EY6A complex (and some particles of this class were also observed to self-associate) (Table 2, Supplementary Fig. 1 and Extended Data Fig. 6a-d). Detailed analysis of this complex led to a reconstruction at a resolution of $3.7 \AA$ (FSC $=0.143, C 1$ symmetry, Extended Data Fig. 7a), which revealed three bound Fabs nestled around the central axis at the top of the spike (Fig. 5a-d). All three RBDs are in an 'up and out' configuration, markedly different to the published open forms (PDB 6VSB and PDB $6 \mathrm{VYB}^{4,6}$ ), being forced to rotate outwards by $\sim 25^{\circ}$, such that the spike is very open and appears on the verge of disruption (Fig. 5e,f). Indeed, the interactions with the Fab must partially stabilize what would otherwise be a disfavored conformation. This fragility is reflected in the observation that arrangement of RBD-EY6A complexes on the top of the spike does not exactly follow three-fold symmetry, with angles between the three RBDs being $120^{\circ}, 119^{\circ}$ and $121^{\circ}$ (although these variations are barely significant). In addition, the orientations of the Vh domains relative to their associated RBDs differ slightly from that of the crystal structure (by $5^{\circ}, 2^{\circ}$ and $7^{\circ}$, respectively). The quality of the density suggests that these are probably samples selected from a continuous distribution.
Many spikes lose structural integrity on EY6A incubation. The majority of the remaining particles form either a roughly two-fold symmetric structure or a triangular association (see Methods, Table 2 and Extended Data Fig. 7b,c). Reconstructions of these particles were anisotropic due to a preferential orientation of the particles on the grid, which was somewhat mitigated by collecting data with a $30^{\circ}$ tilt to yield reconstructions at $4.4 \AA$ and $4.7 \AA$, respectively, in the plane of the grid, but considerably worse resolution perpendicular to the grid (Extended Data Fig. 7b,c). The reconstructions were sufficiently clear to allow the unambiguous fitting of EY6A-RBD complexes (Extended Data Fig. 8a-h) and to confirm that the glycosylation of the spike ectodomain did not significantly alter the mode of association with EY6A. The density for what we assume are the spike $\mathrm{N}$-terminal domains is poor in both reconstructions and we did not attempt to fit a model. These structures probably represent a residual well-structured fragment from the unfolding of the pre-fusion state of the spike. In fact, SDS-PAGE analysis shows that the spike polypeptide remains largely uncleaved (Extended Data Fig. 8i). The 'dimeric' and 'trimeric' structures are formed by different lateral associations and these also differ from that seen for similarly structurally degraded spike-CR3022 complexes $^{14}$. 


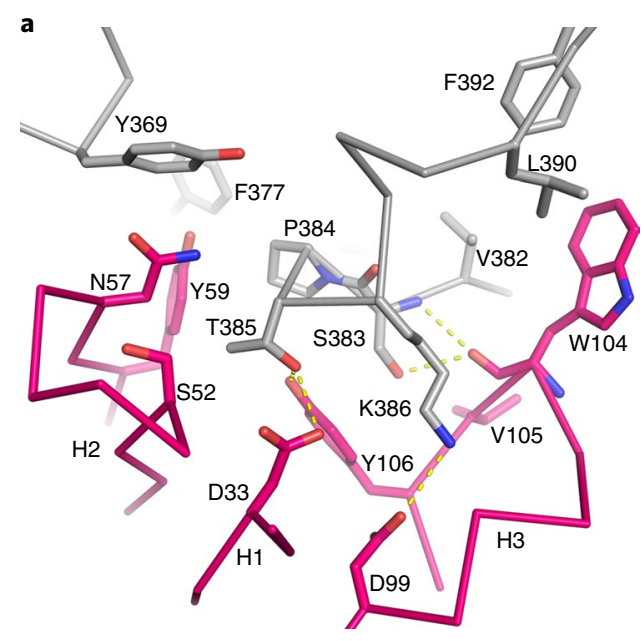

b

c

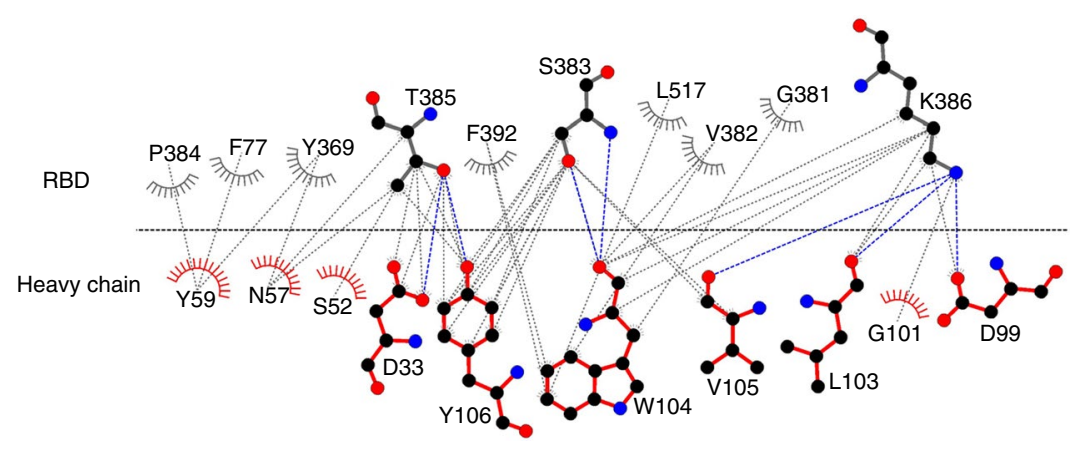

d

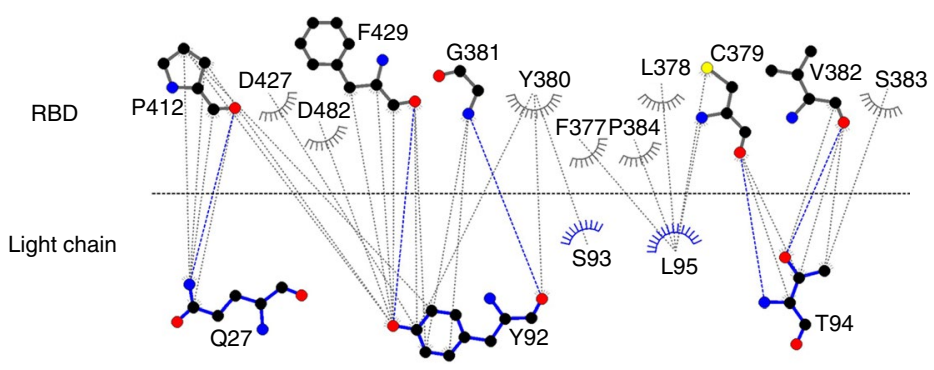

Fig. 4 | Details of RBD-EY6A interactions. a,b, Interactions between the RBD and the heavy chain (a) and between the RBD and the light chain (b). The RBD is shown in gray, the heavy chain in magenta and the light chain in blue. The side chains are drawn in thicker sticks and the main chain C $\alpha$ backbones in thinner sticks. Hydrogen bonds and salt bridges are shown as yellow dashed lines. $\mathbf{c}, \mathbf{d}$, LigPlot ${ }^{30}$ representations showing residues of the RBD involved in direct contacts with the EY6A heavy chain (c) and the light chain (d). Potential hydrogen bonds are shown with blue dotted lines.

\section{Discussion}

Convalescent serum has shown promise in patients severely ill with COVID-1922,23, so immunotherapeutics have potential for treating COVID-19 even at a relatively late stage in the disease. To this end, it is desirable to find a combination of antibodies that neutralize the virus by different mechanisms to mitigate potential immune evasion and antibody-dependent enhancement. One neutralization mechanism is blocking receptor attachment. We propose that the EY6A epitope is another major neutralization target. In support of this proposal, the footprint recognized by EY6A has been reported for several antibodies ${ }^{14,15,24,25}$ and nanobodies ${ }^{20,26}$ raised against SARS-CoV-2, SARS-CoV-1 and MERS. For SARS-CoV-1, CR3022 has also been shown to neutralize synergistically with ACE2-blocking antibodies 9 . Despite the spatial separation of the EY6A and ACE2 footprints, we find some crosstalk between the two binding events and link this to the glycosylation of ACE2 (which is decorated with mannose sugars in the expression system used). Thus, de-glycosylation of ACE2 markedly reduces this crosstalk and we note that this suggests that the neutralization effect of this antibody might vary between different target cells (Extended Data Fig. 3). Because the RBD is a relatively small domain, there are likely to be frequent examples of such an interplay between separate footprints, thus VHH72, which binds an overlapping footprint to EY6A, manages to achieve direct protein-protein clashes with ACE2 by virtue of its different angle of attack ${ }^{20}$.

The EY6A footprint is unusual, because it is completely inaccessible in the pre-fusion spike trimer and bears no N-linked sugars, and indeed we see no effect of RBD glycosylation on EY6A recognition in cryo-EM with glycosylated spike. In the pre-fusion state, the EY6A/CR3022 epitope rests down upon the upper end of the helix-turn-helix between heptad repeat 1 (HR1) and the central helix $(\mathrm{CH})$ of S2, essentially putting a lid on the spring-loaded extension of the helix, which occurs on conversion to the post-fusion state in the vicinity of the mutations designed to prevent conversion between the pre- and post-fusion conformation ${ }^{27}$ (Fig. 6a-c). The residues of the epitope are crucial to these protein-protein 
Table 2 | Cryo-EM data collection, refinement and validation statistics

\begin{tabular}{|c|c|c|c|}
\hline & $\begin{array}{l}\text { Spike-EY6A (EMD-11174, PDB } \\
\text { 6ZDH) }\end{array}$ & $\begin{array}{l}\text { Largely disordered spike-EY6A } \\
\text { trimer (EMD-11173, PDB 6ZDG) }\end{array}$ & $\begin{array}{l}\text { Largely disordered spike-EY6A } \\
\text { dimer (EMD-11184, PDB 6ZFO) }\end{array}$ \\
\hline \multicolumn{4}{|l|}{ Data collection and processing } \\
\hline Electron exposure $\left(\mathrm{e}^{-} / \AA^{2}\right)$ & $42.2(52.5)$ & $42.2(52.5)$ & $42.2(52.5)$ \\
\hline Defocus range $(\mu \mathrm{m})$ & $0.8-2.6$ & $0.8-2.6$ & $0.8-2.6$ \\
\hline Pixel size $(\AA)$ & 0.415 super-resolution & 0.415 super-resolution & 0.415 super-resolution \\
\hline Final particle images (no.) & 144,680 & 41,372 & 119,343 \\
\hline Map resolution $(\AA)$ & 3.7 & $4.7[5.9]$ & 4.4 \\
\hline FSC threshold & 0.5 & 0.5 & 0.5 \\
\hline \multicolumn{4}{|l|}{ Refinement } \\
\hline Initial model used & PDB 6VXX & PDB 6ZCZ & PDB 6ZCZ \\
\hline \multicolumn{4}{|l|}{ Model composition } \\
\hline Non-hydrogen atoms & 34,058 & 14,553 & 9,702 \\
\hline Protein residues & 4,308 & 1,884 & 1,256 \\
\hline Ligands & 49 & 3 & 2 \\
\hline \multicolumn{4}{|l|}{$B$ factors $\left(\AA^{2}\right)$} \\
\hline Protein & 109 & 154 & 82 \\
\hline Ligand & 81 & 168 & 55 \\
\hline \multicolumn{4}{|l|}{ R.m.s. deviations } \\
\hline Bond lengths $(\AA)$ & 0.003 & 0.003 & 0.003 \\
\hline \multicolumn{4}{|l|}{ Ramachandran plot } \\
\hline Favored (\%) & 95.9 & 96.1 & 95.3 \\
\hline Allowed (\%) & 4.1 & 3.9 & 4.7 \\
\hline Disallowed (\%) & 0.02 & 0 & 0 \\
\hline
\end{tabular}

Numbers in brackets refer to the $30^{\circ}$ tilted dataset that was merged with the $0^{\circ}$ data. Square brackets provide values for $\mathrm{Cl}$ symmetry.

interactions, and therefore highly conserved, explaining why mutations that allow SARS-CoV-1 spike to escape binding of CR3022 have not been generated so far ${ }^{9,14}$. EY6A binding to the isolated RBD is tight ( $\sim 2 \mathrm{nM}$, roughly an order of magnitude tighter than CR3022) and the binding pose on top of the spike allows three Fabs to bind simultaneously around the central axis (whereas CR3022 Fab cannot be similarly accommodated). Simple modeling suggests that a similar packing could occur for intact EY6A antibodies (Extended Data Fig. 9).

Binding of EY6A appears to place less strain on the spike than CR3022, because a major portion of spike molecules incubated for $5 \mathrm{~h}$ with EY6A Fab are still in the intact pre-fusion state, with only about one-third being converted. By contrast, we find that by this time point CR3022 has degraded essentially all spikes ${ }^{14}$. In general, we would expect binders at this epitope to neutralize by displacing the 'lid' on the HR1/CH turn, reducing the stability of the pre-fusion state and therefore reducing the barrier to conversion to the more stable post-fusion trimer. In the spike construct we use here, this conversion is hindered by the introduction of proline residues at the turn between the helices. Premature conversion would prevent later attachment to the cell and block infectivity. The kinetics of this process will determine the effectiveness of the antibody in neutralization and ultimately protection from infection. However, as noted above, some binders at this epitope, including EY6A, can also, through direct protein-protein or indirect protein-sugar interactions, interfere with ACE2 attachment. It is possible that the complex dependence of neutralization on spike dynamics might in part explain the marked variation in neutralization titer we observed in PRNT tests, where these assays were performed under different conditions.

In summary, attachment to this single footprint can cause neutralization via more than one mechanism and can exhibit strong 'synergy' with ACE2-blocking antibodies'. Furthermore, due to the high level of conservation of key residues, tight binding antibodies targeting this epitope can neutralize a range of related viruses 

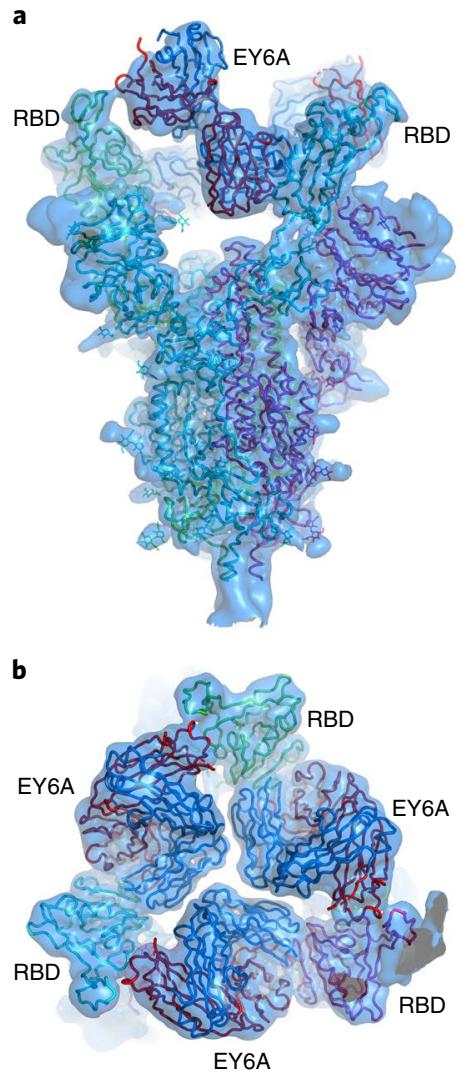

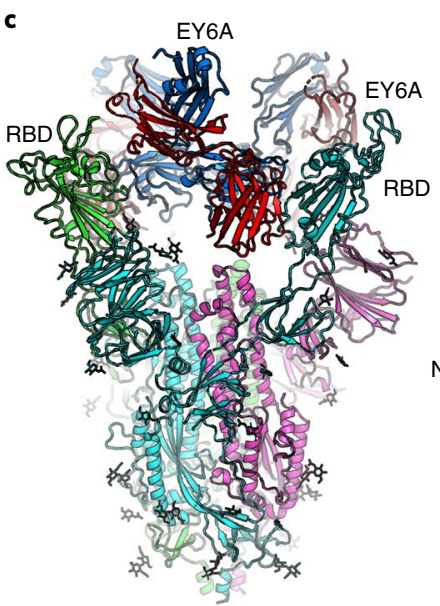

d

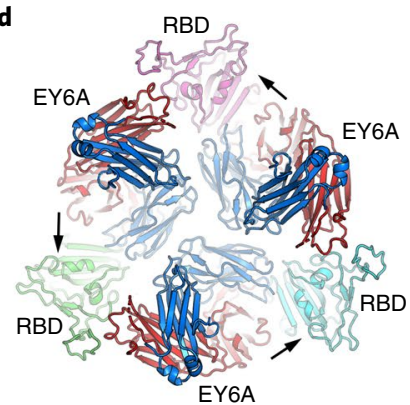

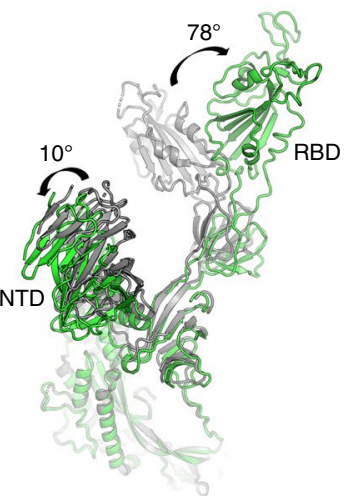

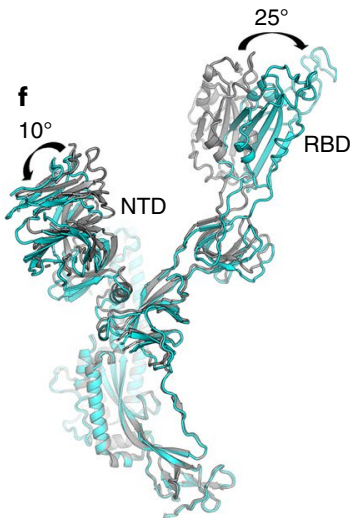

Fig. 5 | EM structure of the SARS-CoV-2 spike-EY6A Fab complex. a,b, Side (a) and top (b) views of the cryo-EM density map drawn as a semi-transparent surface showing three EY6A Fabs bound to the spike. c,d, Side (c) and top (d) views of the overall structure of the spike-EY6A complex. Chains $A, B$ and $C$ of the spike trimer and heavy and light chains of EY6A are shown in green, cyan, magenta, red and blue, respectively. The arrows in $\mathbf{d}$ indicate which RBD each Fab is binding. e,f, Comparison of the EY6A-bound spike structure with a reported open-form spike structure (gray; PDB 6VYB ${ }^{6}$ ) with the A chain in the 'down' conformation (e) and the B chain in the 'up' conformation (f).

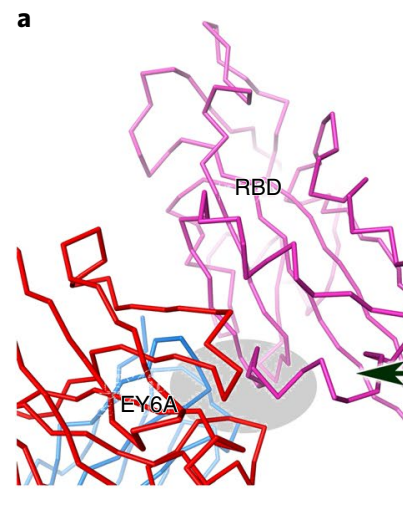

b

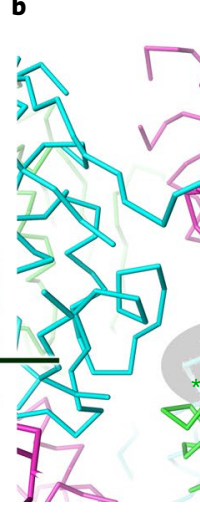

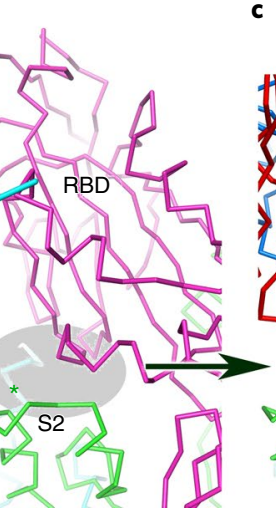

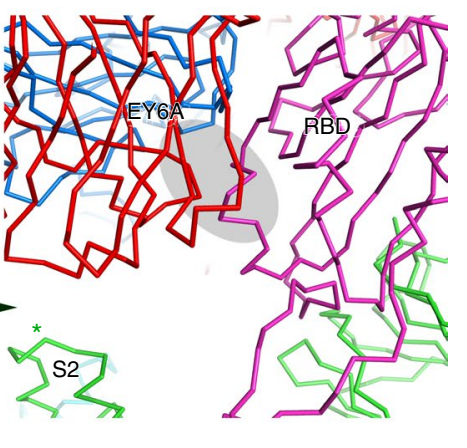

Fig. 6 | EY6A mimics S2 binding to the RBD. a,b, Orientation of the RBD is maintained, demonstrating the commonality of the interaction area between EY6A (a) and the S2 central helix (b). The asterisk marks the position of the double proline mutations that prevent helix extension into the post-fusion state. c, The orientation of S2 is maintained, showing the displacement of the 'lid' from the S2 HR1/CH turn. The gray ellipse on all panels marks the region of the EY6A footprint (the 'lid').

(usually spanning SARS-CoV-1 and SARS-CoV-2 and in some cases extending to MERS). We expect this remarkable epitope to be a major target for therapeutic exploitation.

\section{Online content}

Any methods, additional references, Nature Research reporting summaries, source data, extended data, supplementary information, acknowledgements, peer review information; details of author contributions and competing interests; and statements of data and code availability are available at https://doi.org/10.1038/ s41594-020-0480-y.

Received: 7 June 2020; Accepted: 10 July 2020;

Published online: 31 July 2020 


\section{References}

1. Baud, D. et al. Real estimates of mortality following COVID-19 infection. Lancet Infect. Dis. 20, 773 (2020).

2. Hoffmann, M. et al. SARS-CoV-2 cell entry depends on ACE2 and TMPRSS2 and is blocked by a clinically proven protease inhibitor. Cell $\mathbf{1 8 1}$, 271-280 (2020).

3. Walls, A. C. et al. Tectonic conformational changes of a coronavirus spike glycoprotein promote membrane fusion. Proc. Natl Acad. Sci. USA 114, 11157-11162 (2017).

4. Wrapp, D. et al. Cryo-EM structure of the 2019-nCoV spike in the prefusion conformation. Science 367, 1260-1263 (2020).

5. Song, W., Gui, M., Wang, X. \& Xiang, Y. Cryo-EM structure of the SARS coronavirus spike glycoprotein in complex with its host cell receptor ACE2. PLoS Pathog. 14, e1007236 (2018).

6. Walls, A. C. et al. Structure, function and antigenicity of the SARS-CoV-2 spike glycoprotein. Cell 181, 281-292 (2020).

7. Roy, S. Dynamical asymmetry exposes $2019-\mathrm{nCoV}$ prefusion spike. Preprint at bioRxiv https://www.biorxiv.org/content/10.1101/2020.04.20.052290v1 (2020)

8. Chen, X. et al. Human monoclonal antibodies block the binding of SARS-CoV-2 spike protein to angiotensin converting enzyme 2 receptor. Cell. Mol. Immunol. 17, 647-649 (2020).

9. ter Meulen, J. et al. Human monoclonal antibody combination against SARS coronavirus: synergy and coverage of escape mutants. PLoS Med. 3, e237 (2006).

10. Sui, J. et al. Potent neutralization of severe acute respiratory syndrome (SARS) coronavirus by a human $\mathrm{mAb}$ to $\mathrm{S} 1$ protein that blocks receptor association. Proc. Natl Acad. Sci. USA 101, 2536-2541 (2004).

11. Zhu, Z. et al. Potent cross-reactive neutralization of SARS coronavirus isolates by human monoclonal antibodies. Proc. Natl Acad. Sci. USA 104, 12123-12128 (2007).

12. Ju, B. et al. Human neutralizing antibodies elicited by SARS-CoV-2 infection. Nature https://doi.org/10.1038/s41586-020-2380-z (2020).

13. Shu, Y. \& McCauley, J. GISAID: global initiative on sharing all influenza data-from vision to reality. Euro Surveill. 22, 30494 (2017).

14. Huo, J. et al. Neutralization of SARS-CoV-2 by destruction of the prefusion spike. Cell Host Microbe https://doi.org/10.1016/j.chom.2020.06.010 (2020).

15. Yuan, M. et al. A highly conserved cryptic epitope in the receptorbinding domains of SARS-CoV-2 and SARS-CoV. Science 368, 630-633 (2020).
16. Tian, X. et al. Potent binding of 2019 novel coronavirus spike protein by a SARS coronavirus-specific human monoclonal antibody. Emerg. Microbes Infect. 9, 382-385 (2020).

17. Wang, C. et al. A human monoclonal antibody blocking SARS-CoV-2 infection. Nat. Commun. 11, 2251 (2020).

18. Grist, N. R. Diagnostic Methods in Clinical Virology (Blackwell Scientific, 1966).

19. Huo, J. et al. Neutralizing nanobodies bind SARS-CoV-2 spike RBD and block interaction with ACE2 Nat. Struct. Mol. Biol. https://doi.org/10.1038/ s41594-020-0469-6 (2020).

20. Wrapp, D. et al. Structural basis for potent neutralization of betacoronaviruses by single-domain camelid antibodies. Cell 181, 1004-1015 (2020).

21. Cai, Y. et al. Distinct conformational states of SARS-CoV-2 spike protein. Science https://doi.org/10.1126/science.abd4251 (2020).

22. Bloch, E. M. et al. Deployment of convalescent plasma for the prevention and treatment of COVID-19. J. Clin. Invest. 130, 2757-2765 (2020).

23. Shen, C. et al. Treatment of 5 critically ill patients with COVID-19 with convalescent plasma. JAMA 323, 1582-1589 (2020).

24. Lv, Z. et al. Structural basis for neutralization of SARS-CoV-2 and SARS-CoV by a potent therapeutic antibody. Science https://doi.org/10.1126/science. abc5881 (2020).

25. Okba, N. M. A. et al. Early release-severe acute respiratory syndrome coronavirus 2-specific antibody responses in coronavirus disease. Emerg. Infect. Dis. 26, 1478-1488 (2019).

26. Wu, Y. et al. Identification of human single-domain antibodies against SARSCoV-2. Cell Host Microbe https://doi.org/10.1016/j.chom.2020.04.023 (2020).

27. Pallesen, J. et al. Immunogenicity and structures of a rationally designed prefusion MERS-CoV spike antigen. Proc. Natl Acad. Sci. USA 114, E7348-E7357 (2017).

28. Gong, Y.-N. et al. SARS-CoV-2 genomic surveillance in Taiwan revealed novel ORF8-deletion mutant and clade possibly associated with infections in Middle East. Emerg. Microbes Infect. 9, 1457-1466 (2020).

29. Lan, J. et al. Structure of the SARS-CoV-2 spike receptor-binding domain bound to the ACE2 receptor. Nature 581, 215-220 (2020).

30. Laskowski, R. A. \& Swindells, M. B. LigPlot+: multiple ligand-protein interaction diagrams for drug discovery. J. Chem. Inf. Model. 51, 2778-2786 (2011)

Publisher's note Springer Nature remains neutral with regard to jurisdictional claims in published maps and institutional affiliations.

(c) The Author(s), under exclusive licence to Springer Nature America, Inc. 2020 


\section{Methods}

Antibody isolation study design. This study was designed to isolate SARS-CoV-2 antigen-specific human mAbs from peripheral plasmablasts in humans with natural SARS-CoV-2 infection, to characterize the antigenic specificity and phenotypic activity of SARS-CoV-2 spike-reactive $\mathrm{mAb}$, and to determine the structure of antibody in complex with viral antigen.

The infection of patients/individuals by SARS-CoV-2 was confirmed by positive real-time RT-PCR analyses of respiratory samples, according to the guidelines of the Taiwan Centers for Disease Control (https://www.cdc.gov.tw/En). The study protocol and informed consent were approved by the ethics committee at the Chang Gung Medical Foundation and the Taoyuan General Hospital, Ministry of Health and Welfare, Taiwan. Each patient provided signed informed consent. The study and all associated methods were carried out in accordance with the approved protocol and the Declaration of Helsinki and Good Clinical Practice guidelines.

Sorting of plasmablasts and production of human IgG mAbs. Fresh peripheral blood mononuclear cells (PBMCs) were separated from whole blood by density gradient centrifugation and cryo-preserved PBMCs were thawed. PBMCs were stained with a mix of fluorescent-labeled antibodies to cellular surface markers (all from BD Biosciences) including anti-CD3, anti-CD19, anti-CD27, anti-CD20, anti-CD38, anti-IgG and anti-IgM. Plasmablasts were selected by gating on $\mathrm{CD}^{-}{ }^{-} \mathrm{C}$ $\mathrm{D} 20^{-} \mathrm{CD} 19^{+} \mathrm{CD} 27^{\mathrm{hi}} \mathrm{CD} 38^{\mathrm{h}} \mathrm{IgG}+\mathrm{IgM}^{-}$events and were isolated in chamber as single cells, as previously described ${ }^{31}$. Sorted single cells were used to produce human IgG $\mathrm{mAbs}$ as previously described ${ }^{31}$. Expression vectors that carry variable domains of heavy and light chains were transfected into the 293T cell line for expression of recombinant full-length human IgG monoclonal antibodies in serum-free transfection medium.

To determine the individual gene segments employed by VDJ and VJ rearrangements and the number of nucleotide mutations and amino acid replacements, the variable domain sequences were aligned with germline gene segments using the international ImMunoGeneTics (IMGT) alignment tool (http://www.imgt.org/IMGT_vquest/input).

Protein cloning, expression and purification. All plasmids were sequenced to confirm clones were correct.

EY6A IgG used for neutralization and making Fab. Antibody was expressed using the ExpiCHO expression system (Life Technologies) according to the manufacturer's protocol and purified using a Protein A MabSelect SuRE column (GE Healthcare). The wash buffer contained $20 \mathrm{mM}$ Tris, $150 \mathrm{mM} \mathrm{NaCl}$ buffered to $\mathrm{pH} 8.6$ and the elution was done using $0.1 \mathrm{M}$ citric acid $\mathrm{pH} 2.5$. The eluate was neutralized immediately using $1.5 \mathrm{M}$ Tris $\mathrm{pH} 8.6$ and then buffer-exchanged to PBS using $15 \mathrm{ml} 30-\mathrm{kDa}$ MWCO centrifugal filter (Merck Millipore).

Preparation of Fab-EY6A from IgG. EY6A Fab was digested from IgG with papain using a Pierce Fab Preparation Kit, following the manufacturer's standard protocol

Expression and purification of EY6A-6His Fab. Plasmids encoding the heavy and light chains of EY6A-6His Fab were amplified in Escherichia coli DH5 $\alpha$, then extracted and purified using a Qiagen HiSpeed Plasmid Giga Kit. HEK293T cells were transfected with the two plasmids. The medium was harvested and dialyzed into $1.7 \mathrm{mM} \mathrm{NaH}_{2} \mathrm{PO}_{4}, 23 \mathrm{mM} \mathrm{Na}_{2} \mathrm{HPO}_{4}, 250 \mathrm{mM} \mathrm{NaCl}, \mathrm{pH} 8.0$ at $4^{\circ} \mathrm{C}$ overnight. The sample was then applied to a 5-ml HisTrap nickel column (GE Healthcare). Initially purified EY6A-6His Fab was then loaded onto a Superdex 75 HiLoad $16 / 60$ gel filtration column (GE Healthcare) for further purification using $10 \mathrm{mM}$ HEPES pH 7.4, $150 \mathrm{mM} \mathrm{NaCl}$. Fractions containing EY6A-6His Fab were collected and concentrated.

RBD, ACE2, spike ectodomain and CR3022 cloning. Constructs are as described in ref. ${ }^{14}$.

Nanobody. This was derived from a naive library followed by affinity maturation as described in ref. ${ }^{19}$

Production of RBD and ACE2. Plasmids encoding these constructs were transiently expressed in Expi293 (Thermo Fisher Scientific) and proteins were purified from culture supernatants by immobilized metal affinity chromatography using an automated protocol implemented on an ÄKTAxpress system (GE Healthcare) ${ }^{32}$, followed by size-exclusion chromatography using a Hiload 16/60 Superdex 75 or a Superdex 200 10/300GL column equilibrated in PBS pH 7.4 buffer. Recombinant spike ectodomain was expressed by transient transfection in HEK293S GnTI- cells (ATCC CRL-3022) for nine days at $30^{\circ} \mathrm{C}$. Conditioned medium was dialyzed against $2 \times \mathrm{PBS}$ pH 7.4 buffer. The spike ectodomain was purified by immobilized metal affinity chromatography using Talon resin (Takara Bio) charged with cobalt followed by size-exclusion chromatography using a HiLoad 16/60 Superdex 200 column in $150 \mathrm{mM} \mathrm{NaCl}$, $10 \mathrm{mM}$ HEPES $\mathrm{pH} 8.0,0.02 \% \mathrm{NaN}_{3}$ at $4{ }^{\circ} \mathrm{C}$, before buffer exchange into $2 \mathrm{mM}$ Tris $\mathrm{pH} 8.0,200 \mathrm{mM} \mathrm{NaCl}^{4}$.
De-glycosylation of RBD or ACE2. A $10 \mu \mathrm{l}$ volume of endoglycosidase F1 $\left(\sim 1 \mathrm{mg} \mathrm{ml}^{-1}\right)$ was added to protein $\left(\sim 2 \mathrm{mg} \mathrm{ml}^{-1}, 3 \mathrm{ml}\right)$ and incubated at room temperature for $2 \mathrm{~h}$. The sample was then loaded to a Superdex 75 HiLoad 16/600 gel filtration column (GE Healthcare) for further purification using $10 \mathrm{mM}$ HEPES $\mathrm{pH} 7.4,150 \mathrm{mM} \mathrm{NaCl}$. Purified RBD or ACE2 was concentrated using 10-kDa ultra centrifugal filters (Amicon) to $12 \mathrm{mg} \mathrm{ml}^{-1}$.

Enzyme-linked immunosorbent assay. The ELISA plates (Corning 96-well Clear Polystyrene High Bind Stripwell Microplates) were coated with SARS-CoV-2 antigen (Sino Biological) or SARS antigen (Sino Biological) or Middle East respiratory syndrome coronavirus (MERS) antigen (Sino Biological, 40069-V08B) or human coronavirus OC43 antigen (Sino Biological, 40607-V08B) at optimal concentrations in carbonate buffer and incubated at $4^{\circ} \mathrm{C}$ overnight. The next day, unbound antigens were removed by pipetting to avoid the risk of forming aerosols. Non-specific binding was blocked with a solution of PBS with 3\% BSA at room temperature for $1 \mathrm{~h}$ on a shaker. After removing blocking buffer, mAb-containing cell culture supernatant or purified $\mathrm{mAb}$ preparation was added and incubated at $37^{\circ} \mathrm{C}$ for $1 \mathrm{~h}$. The non-transfected cell culture supernatant, anti-influenza human monoclonal antibody BS 1A (in house), anti-SARS spike monoclonal antibody CR3022 and convalescent serum were used as antibody controls for each experiment. After incubation, the plate was washed and incubated with horseradish peroxidase (HRP)-conjugated rabbit anti-human IgG (Rockland Immunochemicals) as secondary antibody. After incubation, the plate was washed and developed with 3,3',5,5'-tetramethylbenzidine substrate reagent (BD Biosciences). Reaction was stopped by $0.5 \mathrm{M}$ hydrochloric acid and the optical density $(\mathrm{OD})$ was measured at $450 \mathrm{~nm}$ on a microplate reader. The well that yielded an OD value four times the mean absorbance of negative controls (BS 1A) was considered positive.

Immunofluorescence assay. SARS-CoV-2 (strain CDC-4)-infected Vero E6 cells were prepared and fixed with acetone in a Biosafety Level 3 (BSL-3) laboratory following biosafety rules and guidelines ${ }^{28}$. The fixed cells on the cover slips were incubated with anti-SARS-CoV-2 spike EY6A mAb-containing cell culture supernatant or anti-influenza human monoclonal antibody BS $1 \mathrm{~A}$ control (produced in house). Following incubation and washing, the cells were stained with FITC-conjugated goat anti-human IgG secondary antibody (Invitrogen) and Evans Blue dye as counterstain. Binding antibodies were detected by fluorescence microscopy.

Quantitative polymerase chain reaction-based neutralization assay. Neutralization activity of $\mathrm{mAB}$-containing supernatant was measured using a SARS-CoV-2 (strain CDC-4) infection of Vero E6 cells $^{28}$. Briefly, Vero E6 cells were pre-seeded in a 96-well plate at a concentration of $2 \times 10^{4}$ cells per well. On the following day, $\mathrm{mAb}$-containing supernatant was mixed with an equal volume of $100-\mathrm{TCID}_{50}$ virus preparation and incubated at $37^{\circ} \mathrm{C}$ for $1 \mathrm{~h}$. The mixture was added into seeded Vero E6 cells and incubated at $37^{\circ} \mathrm{C}$ for five days. The cell control, virus control and virus back-titration were set up for each experiment. At day 5, the culture supernatant was collected from each well and the viral RNA was extracted by the automatic LabTurbo system (Taigen) following the manufacturer's instructions for the most part, except that the specimen was pretreated with proteinase $\mathrm{K}$ before RNA extraction. Real-time RT-PCR was performed in a $25-\mu$ l reaction containing $5 \mu \mathrm{l}$ of $\mathrm{RNA}^{33}$. The primers and probe used to amplify the $\mathrm{E}$ gene were as follows: E_Sarbeco_F, 5' - ACAGGTACGTTAATAGTTAATAGCGT-3'; E_Sarbeco_R, 5' - ATATTGCAGCAGTACGCACACA-3'; E_Sarbeco_P1, FAM-ACACTAGCCATCCTTACTGCGCTTCG-BBQ.

Cell-based ACE2 or RBD blocking assays. MDCK-SIAT1 cells were stably transfected using a second-generation lentiviral vector, with human ACE2 CDNA or with a construct corresponding to RBD (amino acids 340-538 NITN.GPKK) fused to the transmembrane and cytoplasmic domain of hemagglutinin H7 (A/ HongKong/125/2017) (EPI977395) via a short linker for surface expression (TGSGGSGKLSSGYKDVILWFSFGASCFILLAIVMGLVFICVKNGNMRCTICI*) using the method described above. ACE2-expressing cells were sorted by fluorescent activated cell sorting (FACS), post staining with RBD-6xH, followed by a secondary anti-His AlexaFluor 647 labeled antibody. RBD-expressing cells were FACS-sorted using the CR3022 antibody. Cells $\left(3 \times 10^{4}\right.$ per well) were seeded the day before the assay in flat-bottomed 96 -well plates.

Serial half-log dilutions (ranging from $1 \mu \mathrm{M}$ to $0.1 \mathrm{nM}$ ) of antibodies and controls were performed in $30 \mu \mathrm{l}$ volumes. PBS supplemented with $0.1 \%$ BSA (37525, Thermo Fisher Scientific) was used for dilution of all antibodies. RBD or ACE2-Fc was biotinylated using EZ-link sulfo-NHS-biotin (A39256, Life Technologies). A $30 \mu \mathrm{l}$ volume of biotinylated RBD at $25 \mathrm{nM}$ or Ace2-Fc at $5 \mathrm{nM}$ was added to titrated antibodies. Cells were washed with PBS and $50 \mu \mathrm{l}$ of each mixture of biotinylated protein and antibodies was transferred to the MDCK-ACE2 and incubated for $1 \mathrm{~h}$ at room temperature. Cells were then washed with PBS and incubated for $1 \mathrm{~h}$ with a second layer of streptavidin-HRP antibody (434323, Life Technologies) diluted to 1:1,600 and developed with BM POD substrate (11484281001, Roche) for 5 min before stopping with $1 \mathrm{M} \mathrm{H}_{2} \mathrm{SO}_{4}$. Plates were then read on a ClarioStar Plate Reader. 
Graphs were plotted as percent binding of biotinylated protein (ACE2 or $\mathrm{RBD})$ to its respective ligand on the cell surface. Binding $\%=(X-\min )$ $(\max -\min ) \times 100$ where $X=$ measurement of the competing component, $\min =$ buffer without binder biotinylated protein and $\max =$ biotinylated protein alone. Inhibitory concentration at $50 \%\left(\mathrm{IC}_{50}\right)$ of the antibodies was determined using nonlinear regression [inhibitor] versus a normalized response curve fit using GraphPad Prism 8. Non-biotinylated ACE2-Fc-6H and VHH72-Fc were used as positive controls.

Surface plasmon resonance. SPR experiments were performed using a Biacore T200 system (GE Healthcare). All assays were performed using Sensor Chip protein A (GE Healthcare), with a running buffer of PBS $\mathrm{pH} 7.4$ supplemented with $0.005 \% \mathrm{vol} / \mathrm{vol}$ surfactant P20 (GE Healthcare) at $25^{\circ} \mathrm{C}$. To determine the binding kinetics between the RBD of SARS-CoV-2 and EY6A mAb, two different experimental settings were attempted. The first set-up had RBD-Fc immobilized on the sample flow cell and the reference flow cell was left blank. The EY6A Fab was injected over the two flow cells at a range of five concentrations prepared by serial twofold dilution from $50 \mathrm{nM}$, at a flow rate of $30 \mu \mathrm{lmin}^{-1}$, using a single-cycle kinetics program with an association time of $75 \mathrm{~s}$ and dissociation time of $900 \mathrm{~s}$. Running buffer was also injected using the same program for background subtraction. The second set-up had EY6A IgG immobilized on the sample flow cell and the reference flow cell was left blank. The RBD was injected over the two flow cells at a range of five concentrations prepared by serial twofold dilution from

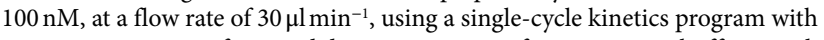
an association time of $90 \mathrm{~s}$ and dissociation time of $60 \mathrm{~s}$. Running buffer was also injected using the same program for background subtraction. All data were fitted to a 1:1 binding model using Biacore T200 Evaluation Software 3.1.

In the competition assay, where either CR3022 IgG or ACE2-hIgG1Fc was used as the ligand, the following samples were injected: (1) a mixture of $1 \mu \mathrm{M}$ EY6A Fab and $0.1 \mu \mathrm{M}$ RBD, (2) a mixture of $1 \mu \mathrm{M}$ (anti-Caspr2) E08R Fab and 0.1 $\mu \mathrm{M}$ RBD (3) $0.1 \mu \mathrm{M}$ RBD, (4) $1 \mu \mathrm{M}$ EY6A Fab and (5) $1 \mu \mathrm{M}$ E08R Fab. In the competition assay where EY6A IgG was used as the ligand, the following samples were injected: (1) a mixture of $1 \mu \mathrm{M}$ CR3022 Fab and $0.1 \mu \mathrm{M}$ RBD, (2) a mixture of $1 \mu \mathrm{M}$ ACE2 and $0.1 \mu \mathrm{M}$ RBD, (3) a mixture of $1 \mu \mathrm{M}$ de-glycosylated ACE2 and $0.1 \mu \mathrm{M} \mathrm{RBD}$, (4) a mixture of $1 \mu \mathrm{M}$ E08R Fab and $0.1 \mu \mathrm{M}$ RBD, (5) $0.1 \mu \mathrm{M}$ RBD, (6) $1 \mu \mathrm{M}$ CR3022 Fab, (7) $1 \mu \mathrm{M}$ ACE2 and (8) $1 \mu \mathrm{M}$ E08R Fab. All injections were performed with an association time of $60 \mathrm{~s}$ and a dissociation time of $600 \mathrm{~s}$. All curves were plotted using GraphPad Prism 8.

Plaque reduction neutralization test (PHE, Porton Down). SARS-CoV-2 (Australia/VIC01/2020) ) $^{34}$ at passage 3 was diluted to a concentration of 933 p.f.u. $\mathrm{ml}^{-1}(70$ p.f.u. $/ 75 \mu \mathrm{l})$ and mixed 50:50 in minimal essential medium (MEM) (Life Technologies) containing 1\% FBS (Life Technologies) and $25 \mathrm{mM}$ HEPES buffer (Sigma) with doubling antibody dilutions in a 96-well V-bottomed plate, repeated in triplicate. The plate was incubated at $37^{\circ} \mathrm{C}$ in a humidified box for $1 \mathrm{~h}$ to allow neutralization to take place before the virus-antibody mixture was transferred into the wells of a twice Dulbecco's PBS-washed 24-well plate containing confluent monolayers of Vero E6 cells (ECACC 85020206; PHE) that had been cultured in MEM containing $10 \%$ (vol/vol) FBS. Virus was allowed to adsorb onto cells at $37^{\circ} \mathrm{C}$ for a further hour in a humidified box, and overlaid with MEM containing $1.5 \%$ carboxymethyl cellulose (Sigma), $4 \%$ (vol/vol) FBS and $25 \mathrm{mM}$ HEPES buffer. After five days of incubation at $37^{\circ} \mathrm{C}$ in a humidified box, the plates were fixed overnight with $20 \%$ formalin/PBS (vol/vol), washed with tap water and then stained with $0.2 \%$ crystal violet solution (Sigma) and plaques were counted. Median neutralizing titers $\left(\mathrm{ND}_{50}\right)$ were determined using the SpearmanKarber formula ${ }^{35}$ relative to virus-only control wells.

Plaque reduction neutralization test (Oxford). Plaque reduction neutralization tests were performed using passage 4 of SARS-CoV-2 Victoria/01/2020 ${ }^{34}$. Virus suspension at appropriate concentrations in DMEM containing 1\% FBS (D1; $100 \mu \mathrm{l})$ was mixed with antibody $(100 \mu \mathrm{l})$ diluted in D1 at a final concentration of $50 \mu \mathrm{g} \mathrm{ml}^{-1}, 25 \mu \mathrm{g} \mathrm{ml}^{-1}, 12.5 \mu \mathrm{g} \mathrm{ml}^{-1}$ or $6.125 \mu \mathrm{g} \mathrm{ml}^{-1}$, in triplicate, in wells of a 24-well tissue culture plate, and incubated at room temperature for $30 \mathrm{~min}$. Thereafter, $0.5 \mathrm{ml}$ of a single-cell suspension of Vero E6 cells in D1 at $5 \times 10^{5} \mathrm{ml}^{-1}$ was added and incubated for $2 \mathrm{~h}$ at $37^{\circ} \mathrm{C}$ before being overlain with $0.5 \mathrm{ml}$ of D1 supplemented with carboxymethyl cellulose (1.5\%). Cultures were incubated for a further four days at $37^{\circ} \mathrm{C}$ before plaques were revealed by staining the cell monolayers with amido black in acetic acid/methanol.

Crystallization, data collection and $\mathrm{X}$-ray structure determination. Purified and de-glycosylated RBD and EY6A Fab were combined in an approximate molar ratio of $1: 1$ at a concentration of $6.5 \mathrm{mg} \mathrm{ml}^{-1}$. $\mathrm{Nb}$ was also combined with EY6A$6 \mathrm{His} \mathrm{Fab}$ and RBD in a 1:1:1 molar ratio with a final concentration of $5.7 \mathrm{mg} \mathrm{ml}^{-1}$. These two complexes were separately incubated at room temperature for $1 \mathrm{~h}$. Initial screening of crystals was performed in Crystalquick 96-well X plates (Greiner Bio-One) with a Cartesian Robot using the nanoliter sitting-drop vapor-diffusion method as previously described ${ }^{36,37}$. Crystals for the binary complex were initially obtained from a Hampton Research Index screen, condition B7 containing $0.04 \mathrm{M}$ $\mathrm{NaH}_{2} \mathrm{PO}_{4}$ and $0.96 \mathrm{M} \mathrm{K}_{2} \mathrm{HPO}_{4}$ and further optimized to produce better crystals in
$0.02 \mathrm{M} \mathrm{NaH}_{2} \mathrm{PO}_{4}$ and $0.98 \mathrm{M} \mathrm{K}_{2} \mathrm{HPO}_{4}$. Good crystals for the ternary complex were also obtained from the Index screen condition G1 containing 25\% (wt/vol) PEG 3350, 0.2 $\mathrm{M} \mathrm{NaCl}$ and $0.1 \mathrm{M}$ Tris $\mathrm{pH} 8.5$.

Crystals were soaked in a solution containing $25 \%$ glycerol and a $75 \%$ reservoir solution for a few seconds and then mounted in loops and frozen in liquid nitrogen before data collection. Diffraction data were collected at $100 \mathrm{~K}$ at Beamline I03 (wavelength 0.97625 ) at the Diamond Light Source. Diffraction images of $0.1^{\circ}$ rotation were recorded on an Eiger $2 \mathrm{XE} 16 \mathrm{M}$ detector with an exposure time of $0.01 \mathrm{~s}$ per frame, beam size of $80 \times 20 \mu \mathrm{m}$ and $100 \%$ beam transmission. Data were indexed, integrated and scaled with the automated data-processing program Xia2-dials ${ }^{38,39}$. The binary complex structure (Table 1) was determined by molecular replacement with PHASER ${ }^{40}$ using search models of antibody CR3022 Fab and the RBD of the RBD-CR3022 Fab complex (PDB 6YLA ${ }^{14}$ ). There are three RBD-EY6A complexes in the crystal asymmetric unit, resulting in a crystal solvent content of $\sim 75 \%$. For the ternary complex, data were collected on Beamline I03 with an exposure time of $0.008 \mathrm{~s}$ per $0.1^{\circ}$ frame, beam size of $80 \times 20 \mu \mathrm{m}$ and $100 \%$ beam transmission. There is one RBD-EY6A-Nb complex in the asymmetric unit and a solvent content of $\sim 61 \%$.

$\mathrm{X}$-ray crystallographic refinement and electron density map generation. One cycle of REFMAC $5^{41}$ was used to refine atomic coordinates after manual correction in $\mathrm{COOT}^{42}$ to the protein sequence from the search model. For both the binary and ternary complexes, the final refinement used PHENIX ${ }^{43}$. There is well-ordered density for a single glycan at glycosylation site N343 in the RBD. Data collection and structure refinement statistics are provided in Table 1.

\section{EY6A Fab-spike complex preparation and cryo-electron microscopy data} collection. Following size-exclusion chromatography purification, spike protein was buffer-exchanged into $2 \mathrm{mM}$ Tris $\mathrm{pH} 8.0,200 \mathrm{mM} \mathrm{NaCl}, 0.02 \% \mathrm{NaN}_{3}$ buffer using a desalting column (Zeba, Thermo Fisher). A final concentration of $0.18 \mathrm{mg} \mathrm{ml}^{-1}$ was incubated with EY6A Fab (in the same buffer) in a 6:1 molar ratio (Fab to trimeric spike) at room temperature for $5 \mathrm{~h}$. Control grids of spike alone after incubation at room temperature for $5 \mathrm{~h}$ were also prepared.

Each grid was prepared using a $3-\mu \mathrm{l}$ sample applied to a freshly glow-discharged (on high for $20 \mathrm{~s}$; Plasma Cleaner PDC-002-CE, Harrick Plasma) holey carbon-coated 200-mesh copper grid (C-Flat, CF-2/1, Protochips) and excess liquid was removed by blotting for $5-5.5 \mathrm{~s}$ with a blotting force of -1 using Vitrobot filter paper (grade 595, Ted Pella) at $4.5^{\circ} \mathrm{C}$ and $100 \%$ relative humidity, then it was immediately plunge-frozen in ethane slush using a Vitrobot Mark IV (Thermo Fisher).

Grids were screened on a Titan Krios microscope using the SerialEM program, operating at $300 \mathrm{kV}$ (Thermo Fisher). Videos were collected on a K3 detector on the Titan Krios microscope operating at $300 \mathrm{kV}$ in super-resolution mode, with a calibrated super-resolution pixel size of $0.415 \AA$ per pixel at both $0^{\circ}$ and $30^{\circ}$ tilt. To compensate for the poorer contrast with tilted data, it was necessary to use a higher dose rate for the latter dataset.

Cryo-electron microscopy data processing. Alignment and motion correction were performed using Relion3.1's implementation of motion correction ${ }^{44}$, with a five-by-five patch-based alignment. All frames were binned by two, resulting in a final calibrated pixel size of $0.83 \AA$ per pixel. The contrast transfer function (CTF) of full-dose and non-weighted micrographs was estimated within a CryoSPARC wrapper for Gctf-v1.06 ${ }^{45}$. Images were then manually inspected and those with poor CTF fits were discarded. Particles were then picked by unbiased blob-picking in CryoSPARC v.2.14.1 $1^{46}$ and subjected to rounds of $2 \mathrm{D}$ classification.

For the spike-EY6A dataset (structure A), 2,096,246 spike-like particles were used to make a template to pick particles from the untilted dataset, which were then filtered by 2D classification to 110,096 particles and then further refined by $3 \mathrm{D}$ classification with an ab initio model set. For the $30^{\circ}$ dataset, 124,194 particles were used as a template and filtered by $2 \mathrm{D}$ classification to a set of 84,230 particles and then, as before, further refined by unbiased 3D classification. The two particle sets were then refined together, with a final set of 144,680 particles.

For $\mathrm{B}$ and $\mathrm{C}$ (triangular ring and 'dimeric' form), particles from both the zero and $30^{\circ}$ datasets were combined in a similar manner to the spike-EY6A dataset using the 'Exposure Group Utilities' module in CryoSPARC. Both particle sets (B, 41,372 particles and C, 119,343 particles) were then reclassified and the best class refined with non-uniform refinement. For B, C3 symmetry was imposed at this final refinement stage, resulting in an appreciable improvement in resolution, as indicated by inspection and the gold-standard FSC $=0.143$ (4.7 versus $5.9 \AA$, see Table 2).

Cryo-electron microscopy model building and refinement. The electron microscopy density of spike-EY6A was fitted with the structure of a closed form of spike (PDB 6VXX'), apart from the RBDs and EY6A Fab, which were fitted with RBD-EY6A of the ternary crystal structure using COOT $^{42}$. Because of the lower resolution, RBD and EY6A are only fitted to the 'dimeric' and 'trimeric' electron microscopy density. The spike-EY6A structure was refined with PHENIX ${ }^{4}$ real-space refinement, first as a rigid body and then by positional and B-factor refinements. Only rigid body refinement was applied to the 'dimeric' and 'trimeric' 
complexes. The statistics of EM data collection and structure refinement are shown in Table 2 .

Illustrations and figures. Structural comparisons used $\mathrm{SHP}^{47}$, residues forming the $\mathrm{RBD} / \mathrm{Fab}$ interface were identified with PISA ${ }^{48}$, figures were prepared with PyMOL (The PyMOL Molecular Graphics System, Version 1.2r3pre, Schrödinger). Sequence alignments were generated using Clustal Omega ${ }^{49}$ and colored with ESPript ${ }^{50}$.

Reporting Summary. Further information on research design is available in the Nature Research Reporting Summary linked to this article.

\section{Data availability}

All the data supporting the findings of this study are available within the paper, Extended Data and Supplementary Information files. The coordinates and structure factors for SARS-CoV-2 RBD-EY6A and SARS-CoV-2 RBD-EY6A$\mathrm{Nb}$ have been deposited in the wwPDB with accession codes 6ZER and 6ZCZ, respectively. Electron microscopy maps and structure models are deposited in the EMDB and PDB under accession codes EMD-11174 and PDB 6ZDH (spikeEY6A), EMD-11184 and PDB 6ZFO and EMD-11173 and PDB 6ZDG (the dimeric and trimeric complexes of the largely structurally disordered spike ectodomain with bound EY6A Fab, respectively). Requests for antibody material should be addressed to K.-Y.A.H.

\section{References}

31. Arthur Huang, K.-Y. et al. Epitope-associated and specificity-focused features of EV71-neutralizing antibody repertoires from plasmablasts of infected children. Nat. Commun. 8, 762 (2017).

32. Nettleship, J. E., Rahman-Huq, N. \& Owens, R. J. The production of glycoproteins by transient expression in mammalian cells. Methods Mol. Biol. 498, 245-263 (2009).

33. Corman, V. M. et al. Detection of 2019 novel coronavirus (2019-nCoV) by real-time RT-PCR. Euro Surveill. 25, 2000045 (2020).

34. Caly, L. et al. Isolation and rapid sharing of the 2019 novel coronavirus (SAR-CoV-2) from the first patient diagnosed with COVID-19 in Australia. Med. J. Aust. 212, 459-462 (2020).

35. Kärber, G. Beitrag zur kollektiven behandlung pharmakologischer reihenversuche. Naunyn. Schmiedebergs. Arch. Exp. Pathol. Pharmakol. 162, 480-483 (1931).

36. Walter, T. S. et al. A procedure for setting up high-throughput nanolitre crystallization experiments. I. Protocol design and validation. J. Appl. Crystallogr. 36, 308-314 (2003).

37. Walter, T. S. et al. A procedure for setting up high-throughput nanolitre crystallization experiments. Crystallization workflow for initial screening, automated storage, imaging and optimization. Acta Crystallogr. D Biol. Crystallogr. 61, 651-657 (2005)

38. Winter, G. Xia2: an expert system for macromolecular crystallography data reduction. J. Appl. Crystallogr. 43, 186-190 (2010).

39. Winter, G. et al. DIALS: implementation and evaluation of a new integration package. Acta Crystallogr. D Struct. Biol. 74, 85-97 (2018).

40. McCoy, A. J. et al. Phaser crystallographic software. J. Appl. Crystallogr. 40, 658-674 (2007).

41. Murshudov, G. N. et al. REFMAC5 for the refinement of macromolecular crystal structures. Acta Crystallogr. D Biol. Crystallogr. 67, 355-367 (2011).

42. Emsley, P. \& Cowtan, K. Coot: model-building tools for molecular graphics. Acta Crystallogr. D Biol. Crystallogr. 60, 2126-2132 (2004).

43. Liebschner, D. et al. Macromolecular structure determination using X-rays, neutrons and electrons: recent developments in Phenix. Acta Crystallogr. D Struct. Biol. 75, 861-877 (2019).

44. Zivanov, J. et al. New tools for automated high-resolution cryo-EM structure determination in RELION-3. Elife 7, e42166 (2018).

45. Zhang, K. Gctf: real-time CTF determination and correction. J. Struct. Biol. 193, 1-12 (2016)

46. Punjani, A., Rubinstein, J. L., Fleet, D. J. \& Brubaker, M. A. CryoSPARC: algorithms for rapid unsupervised cryo-EM structure determination. Nat. Methods 14, 290-296 (2017).

47. Stuart, D. I., Levine, M., Muirhead, H. \& Stammers, D. K. Crystal structure of cat muscle pyruvate kinase at a resolution of $2.6 \AA$. J. Mol. Biol. 134, 109-142 (1979).
48. Krissinel, E. \& Henrick, K. Inference of macromolecular assemblies from crystalline state. J. Mol. Biol. 372, 774-797 (2007).

49. Sievers, F. \& Higgins, D. G. Clustal Omega for making accurate alignments of many protein sequences. Protein Sci. 27, 135-145 (2018).

50. Gouet, P., Robert, X. \& Courcelle, E. ESPript/ENDscript: extracting and rendering sequence and 3D information from atomic structures of proteins. Nucleic Acids Res. 31, 3320-3323 (2003).

\section{Acknowledgements}

We acknowledge the BD FACSAria cell sorter service provided by the Core Instrument Center of Chang Gung University. The sorting of plasmablasts and production and characterization of human mAbs were supported by the Chang Gung Memorial Hospital (BMRPE22). This work was supported by the Chinese Academy of Medical Sciences (CAMS) Innovation Fund for Medical Science (CIFMS), China (grant no. 2018-I2M-2002) to D.I.S., G.R.S. and A.R.T., which supported P.R.; E.E.F., H.M.E.D. and J. Ren are supported by the Wellcome Trust (101122/Z/13/Z); Y.Z. by Cancer Research UK (C375/ A17721); and D.I.S. and E.E.F. by the UK Medical Research Council (MR/N00065X/1); J.H. is supported by a grant from the EPA Cephalosporin Fund; and PPUK is funded by the Rosalind Franklin Institute EPSRC grant no. EP/S025243/1 (J.H.N. and R.J.O.). The National Institute for Health Research Biomedical Research Centre Funding Scheme supports G.R.S. We are also grateful for a Fast Grant from Fast Grants (Mercatus Center) to support the isolation of human monoclonal antibodies to SARS-2. G.R.S. is also supported as a Wellcome Trust Senior Investigator (grant no. 095541/A/11/Z). T.K.T. is funded by an EPA Cephalosporin Early Career Teaching and Research Fellowship and the Townsend-Jeantet Charitable Trust (charity no. 1011770). T.M. is supported by Cancer Research UK grants C20724/A14414 and C20724/A26752 to C. Siebold. This is a contribution from the UK Instruct-ERIC Centre. The Wellcome Centre for Human Genetics is supported by the Wellcome Trust (grant no. 090532/Z/09/Z). Virus used for the neutralization assays was a gift from J. Druce at the Doherty Centre, Melbourne, Australia. We acknowledge the Diamond Light Source for time on Beamline I03 under proposal mx19946 and for electron microscope time at the UK National Electron Bio-Imaging Centre (eBIC), proposal BI26983, both COVID-19 Rapid Access. D.I.S. is a Jenner Investigator. We thank the teams, especially at the Diamond Light Source and Department of Structural Biology, Oxford University, that have enabled work to continue during the pandemic.

\section{Author contributions}

J.H. performed interaction analyses and T.K.T., P.R., R.F.D., A.R.T., K.B., K.G., J.T., M.W.C., J.G.J., M.K., W.J. and A.R.T. prepared material for and analyzed and executed neutralization assays and cell-based ACE2-blocking assays. Y.Z., D.Z., J.H. and J. Ren performed sample preparation for crystallographic experiments and processed the data. N.G.P. assisted with X-ray diffraction data collection. J. Ren refined the structures and, together with E.E.F. and D.I.S., analyzed the results. G.R.S., J.M. and P.S. prepared the spike construct. L.C. helped perform cryo-EM data processing. T.M. prepared the spike sample. H.M.E.D., R.R.R. and P.N.M.S. prepared cryo-EM grids. H.M.E.D. performed cryo-EM sample preparation, screening and processing. J. Radecke performed cryo-EM data collection and J. Ren refined the cryo-EM structures. E.E.F., J. Ren, Y.Z., K.-Y.A.H. and D.I.S. wrote the manuscript in discussion with R.J.O and J.H.N. K.-Y.A.H. isolated and characterized EY6A. C.-P.C., C.-G.H., T.-H.C., S.-R.S., Y.-C.L., C.-Y.C., S.-H.C., Y.-C.H., T.-Y.L. and C.M. helped prepare materials, perform experiments and analyze data. All authors read and approved the manuscript.

\section{Competing interests}

K.-Y.A.H. has filed a patent application (US Provisional Application Serial No. $63 / 029,980)$ on antibody EY6A. The other authors declare no competing interests.

\section{Additional information}

Extended data is available for this paper at https://doi.org/10.1038/s41594-020-0480-y. Supplementary information is available for this paper at https://doi.org/10.1038/ s41594-020-0480-y.

Correspondence and requests for materials should be addressed to D.I.S. or K.-Y.A.H. Peer review information Peer reviewer reports are available. Inês Chen was the primary editor on this article and managed its editorial process and peer review in collaboration with the rest of the editorial team.

Reprints and permissions information is available at www.nature.com/reprints. 
ARTICLES

NATURE STRUCTURAL \& MOLECULAR BIOLOGY

a

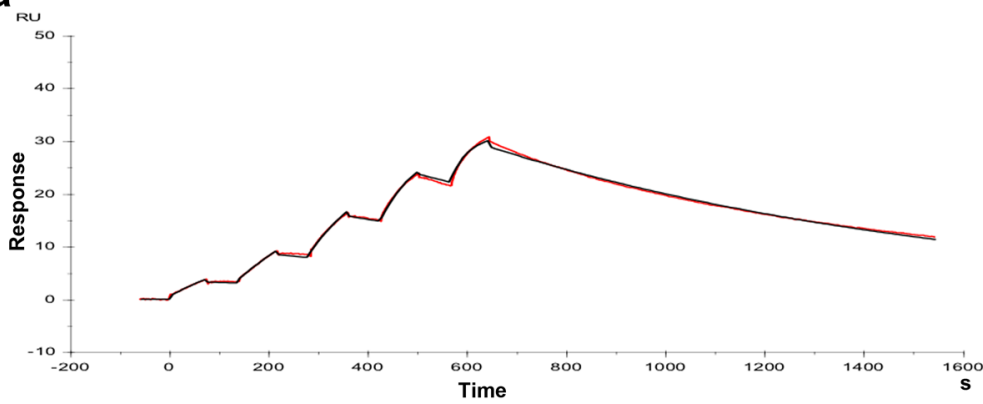

b

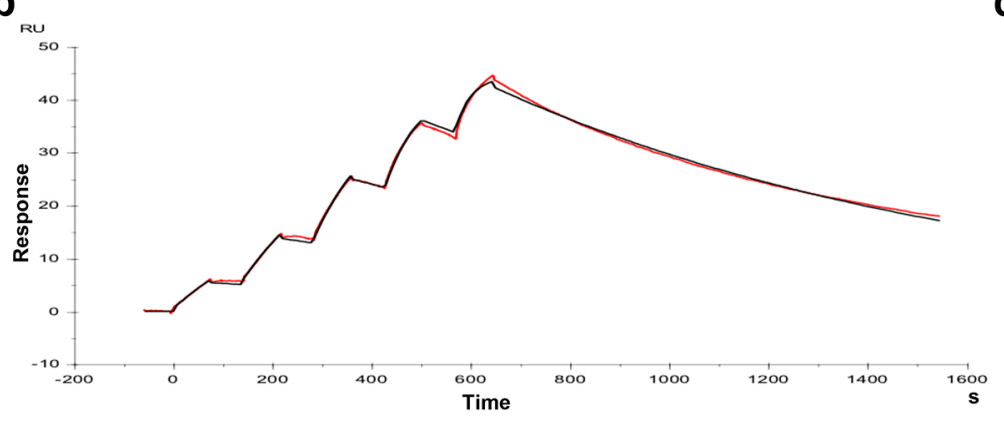

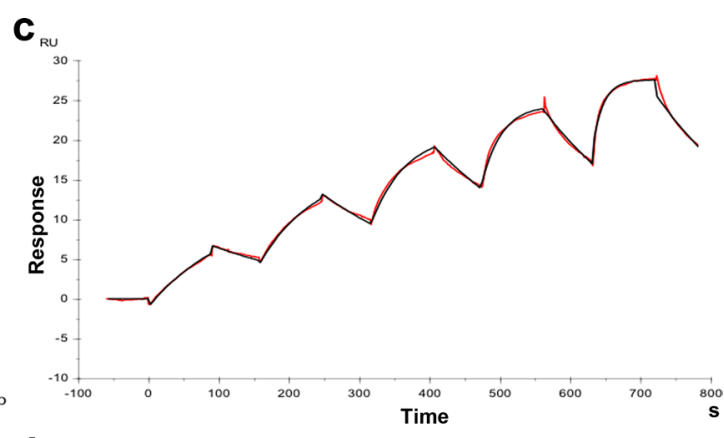

d

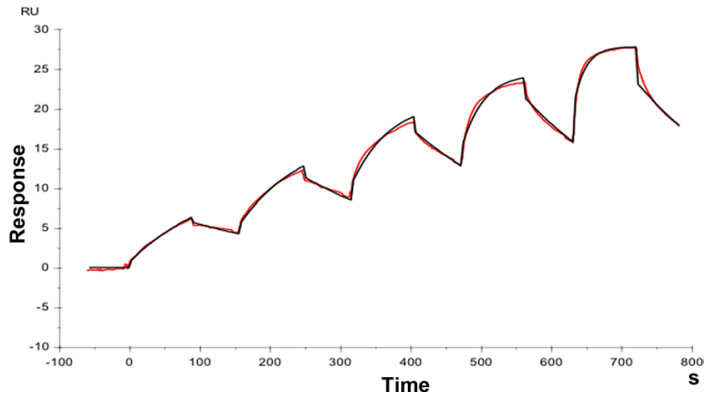

Extended Data Fig. 1 | Binding affinity between RBD and EY6A Fab. a, b, Surface plasmon resonance binding sensorgrams. a, RBD-Fc was immobilized as the ligand and EY6A Fab was used as analyse at five concentrations (3.125, 6.25, 12.5, 25 and $50 \mathrm{nM})$. b, EY6A lG was immobilised as the ligand and RBD was used as analyze at five concentrations $(6.25,12.5,2550$ and $100 \mathrm{nM})$. The average kinetic values from these two sets of experiment are listed in Supplementary Table 1.

NATURE STRUCTURAL \& MOLECULAR BIOLOGY | www.nature.com/nsmb 
a

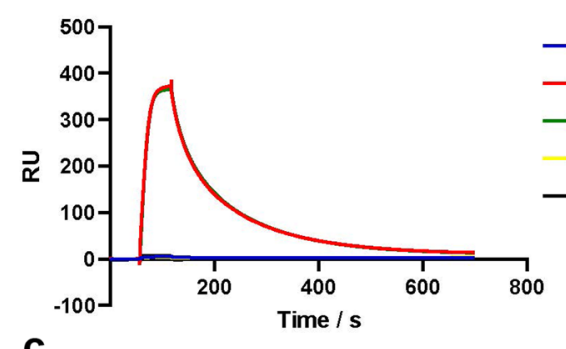

C

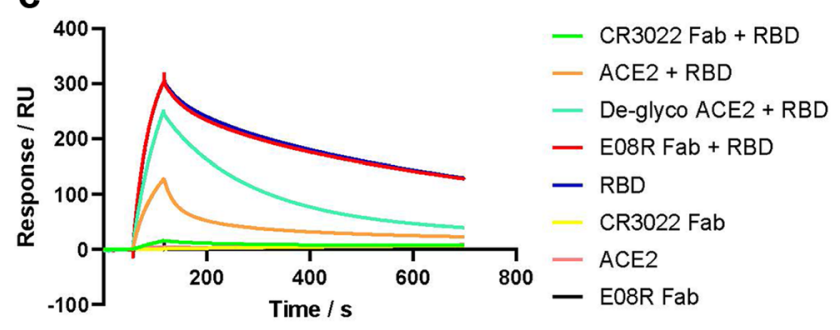

d

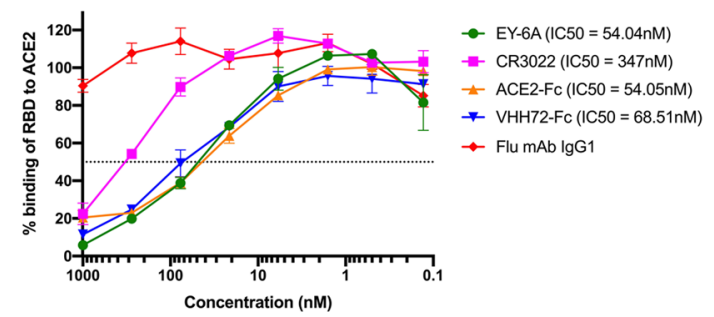

b

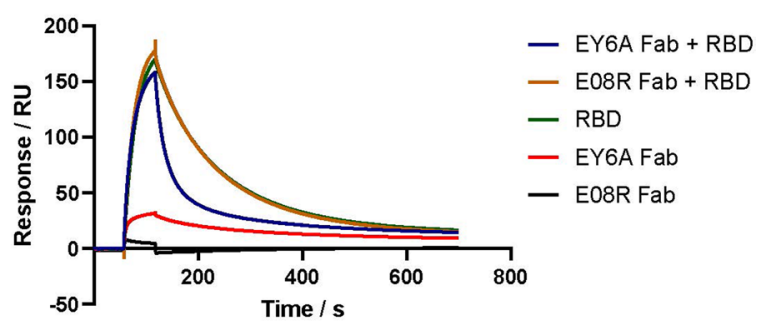

e

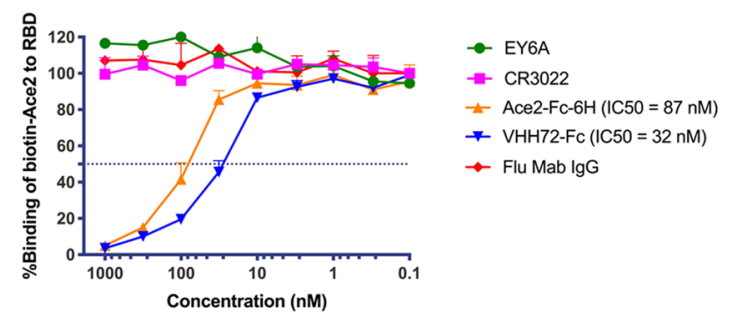

Extended Data Fig. 2 | Binding competition of EY6A and ACE2 for RBD. Surface plasmon resonance binding sensorgrams. a, CR3022 IgG was immobilised, the following samples were injected: (1) mixed $1 \mu \mathrm{M} \mathrm{EY6A} \mathrm{Fab} \mathrm{and} 0.1 \mu \mathrm{M}$ RBD; (2) mixed $1 \mu \mathrm{M}$ E08R Fab and $0.1 \mu \mathrm{M}$ RBD; (3) $0.1 \mu \mathrm{M}$ RBD; (4) $1 \mu \mathrm{M}$ EY6A Fab; (5) $1 \mu \mathrm{M}$ E08R Fab. b, ACE2-hlgG1Fc was immobilised, the following samples were injected: (1) mixed $1 \mu \mathrm{M} \mathrm{EY6A} \mathrm{Fab} \mathrm{and} \mathrm{0.1 \mu M} \mathrm{RBD;}$ (2) mixed $1 \mu \mathrm{M}$ E08R Fab and $0.1 \mu \mathrm{M}$ RBD; (3) $0.1 \mu \mathrm{M} \mathrm{RBD;} \mathrm{(4)} 1 \mu \mathrm{M}$ EY6A Fab; (5) $1 \mu \mathrm{M}$ E08R Fab. c, EY6A IgG was immobilised, the following samples were injected: (1) mixed $1 \mu \mathrm{M}$ CR3022 Fab and $0.1 \mu \mathrm{M}$ RBD, (2) mixed $1 \mu \mathrm{M}$ ACE2 and $0.1 \mu \mathrm{M}$ RBD, (3) mixed $1 \mu \mathrm{M}$ de-glycosylated ACE2 and $0.1 \mu \mathrm{M}$ RBD, (4) mixed $1 \mu \mathrm{M}$ E08R Fab and $0.1 \mu \mathrm{M} \mathrm{RBD}$, (5) $0.1 \mu \mathrm{M} \mathrm{RBD,} \mathrm{(6)} 1 \mu \mathrm{M}$ CR3022 Fab, (7) $1 \mu \mathrm{M} \mathrm{ACE2,} \mathrm{(8)} 1 \mu \mathrm{M}$ E08R Fab. d, Purified antibodies or ACE2-Fc were added to biotin labeled RBD-6H ( $25 \mathrm{nM}$ ) and added to MDCK-SIAT1 cells stably expressing human ACE2 on the cell surface (MDCK-ACE2). The amount of biotinylated RBD bound to the cell was measured. Experiments were performed in duplicate and the mean and s.d. values are shown. IC $C_{50}$ is calculated as described in Methods. e, ACE2-Fc and nanobody VHH72-Fc were mixed with biotin-labeled ACE2-Fc ( $5 \mathrm{nM}$ ) and added to MDCK-SIAT1 cells stably expressing RBD. The percentage of biotin-ACE2-Fc bound to RBD was measured. Experiments were performed in duplicate and the mean and s.d. values are shown. $I C_{50}$ is calculated as described in Methods. 

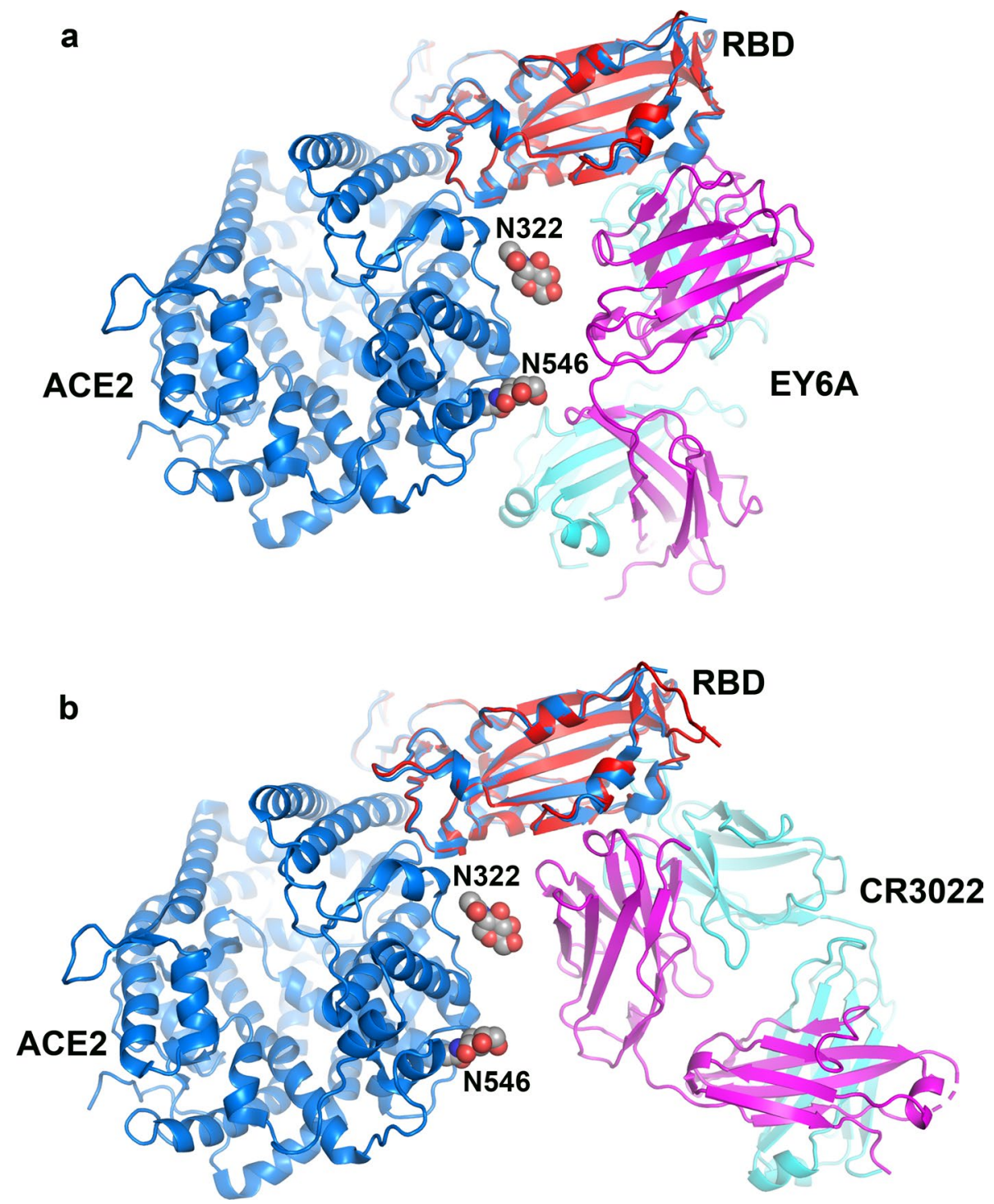

Extended Data Fig. 3 | Glycans may modulate interactions between ACE2 and EY6A. a, EY6A (magenta and cyan cartoon depiction) is shown bound to Spike RBD (red cartoon unbound structure overlaying blue cartoon, bound to ACE2). The sugars on ACE2 at N322 and N546 are shown in space filling representation and approach closer to EY6A than CR3022 shown in $\mathbf{b}$ 

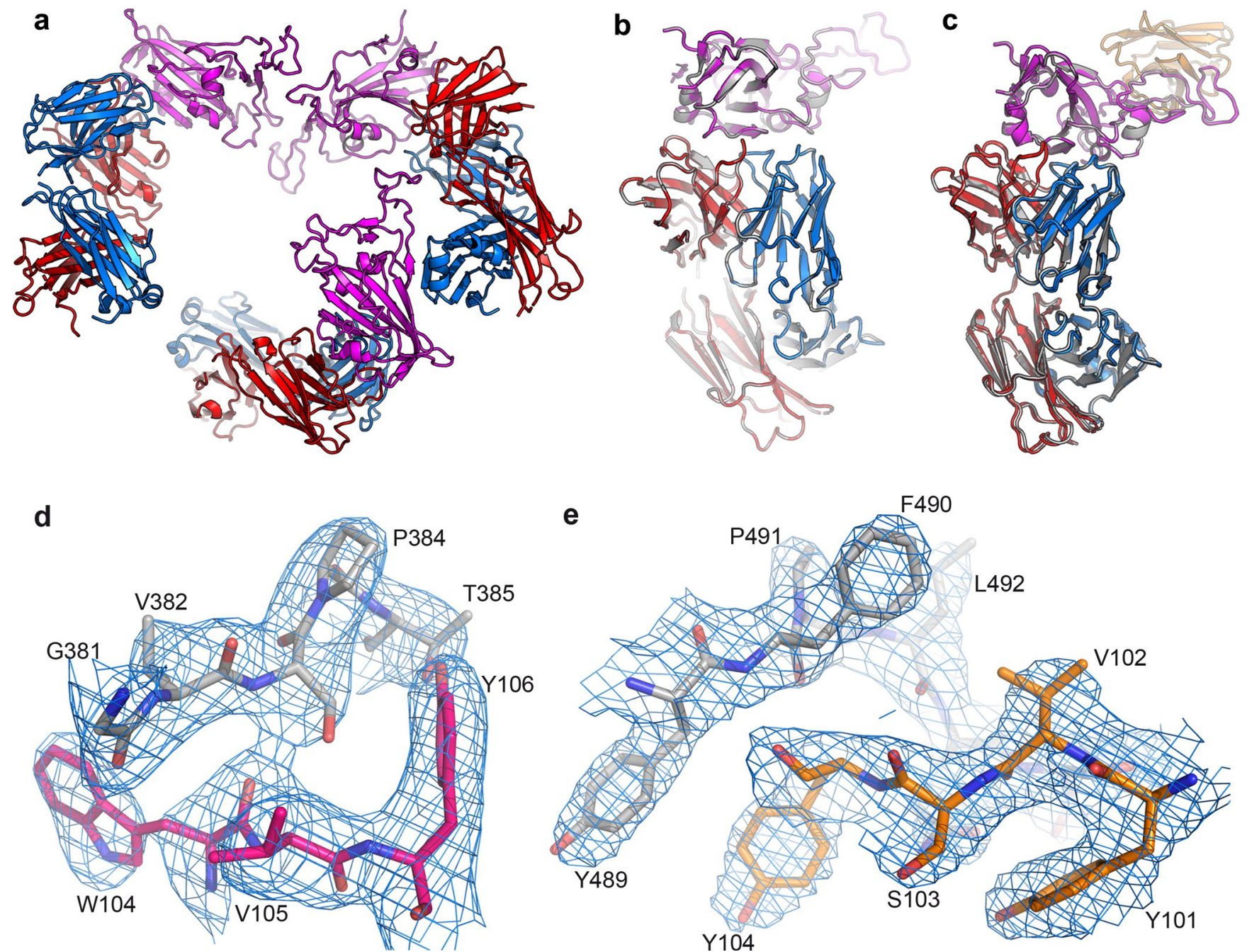

Extended Data Fig. 4 | Comparison of the binding poses in crystal structures of the binary and ternary complexes. a, Three RBD-EY6A binary complexes in the crystal asymmetric unit. RBD is shown in magenta, EY6A heavy chain in red and light chain in blue. $\mathbf{b}$, Superimposition of the three RBD-EY6A complexes in the asymmetric unit showing the same binding pose. One complex is colored as in (a) and the other two in gray. c, Comparison of RBDEY6A binary complex with RBD-EY6A-Nb ternary complex by overlapping the RBDs. The RBD and EY6A are colored as in (a), with Nb in orange in the ternary complex, and gray in the binary complex. d, e show the electron density for the RBD-EY6A-Nb ternary crystal structure. In (d) the RBD is shown as gray sticks and EY6A heavy chain as red sticks. In (e) the RBD is in gray and the Nb in orange. The density is contoured at $1.2 \sigma$. 
a

$\alpha 1$

$\stackrel{\beta 1}{\longrightarrow} \quad \stackrel{\alpha 2}{\longrightarrow}$ elee

$\stackrel{\beta 2}{\longrightarrow} \stackrel{\alpha 3}{\longrightarrow}$

SARS-CoV-2 333 TNLCPFGEVFNATRFA SVYAWNRRRI SNCVADYSVLYNSA SFSTFKCYGVSPTKLNDLCF SARS-COV TNLCPEGEVFNATKEP SVYAWERKKI SNCVADYSVLYNSTFESTFKCYGVSATKLNDLCF $\underset{E Y G A}{\mathrm{CR} 3022} \rightarrow$

$\beta 3 \quad \alpha 4$

$\alpha 5$

$\bullet: 8 \bullet: 8: 8: 8: 8: 8: 8: \bullet \bullet \bullet \bullet$

SARS-CoV-2 393 TNVYADSFVIRGDEVRQIAPGQTGKIADYNYKLPDDFTGCVIAWNSNNLDSKVGGNYNYL

34

$\alpha 6$ SARS-COV SNVYADSFVVKGDDVRQIAPGQTGVIADYNYKLPDDFMGCVIAWNTRNIDATSTGNYNYK

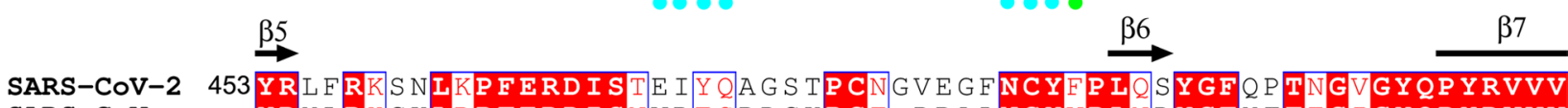

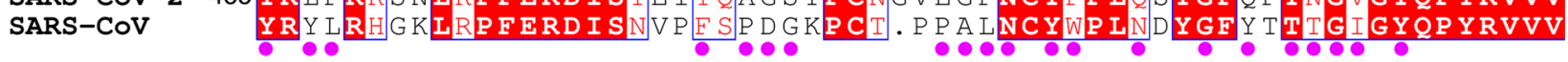
$\begin{array}{ll}\text { SARS-CoV-2 } & \begin{array}{l}\longrightarrow \\ \text { LSFELIHAPATVCG } \\ \text { SARS-COV }\end{array} \\ \text { LSFELINAPATVCG }\end{array}$

b

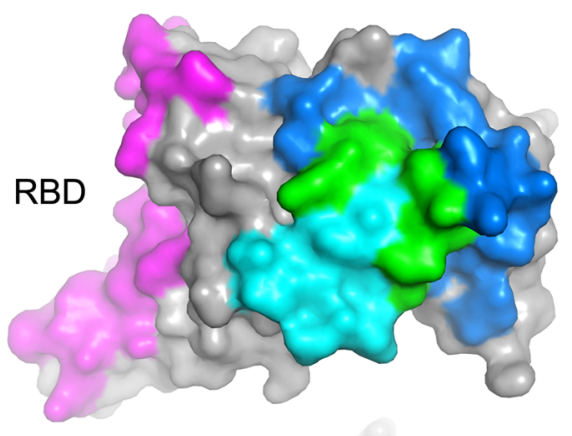

C

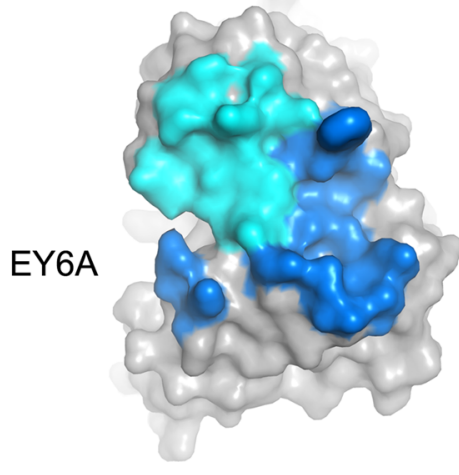

$\mathbf{f}$

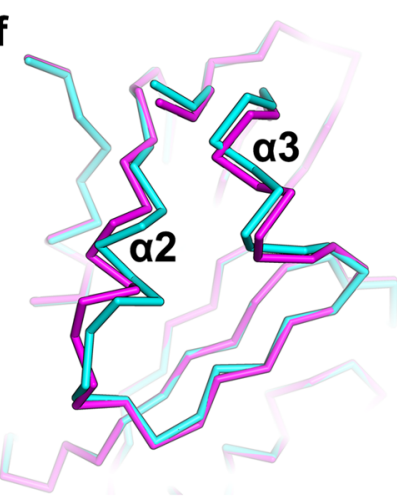

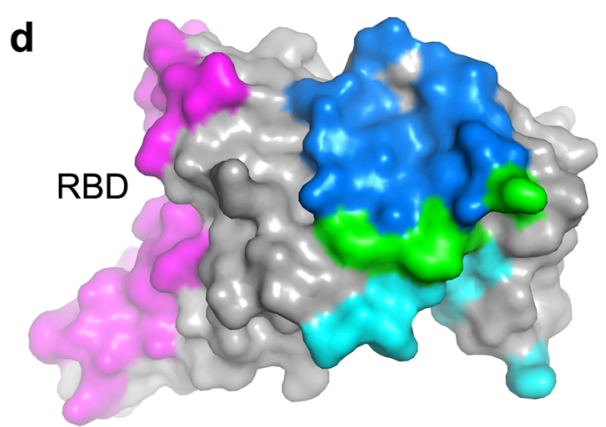

e
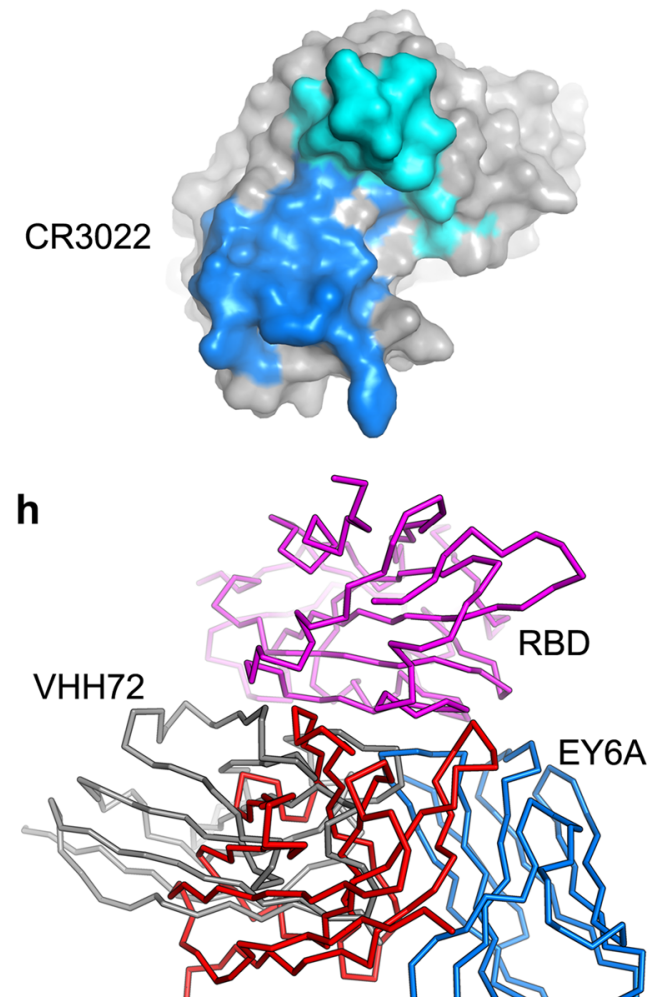

Extended Data Fig. 5 | See next page for caption.

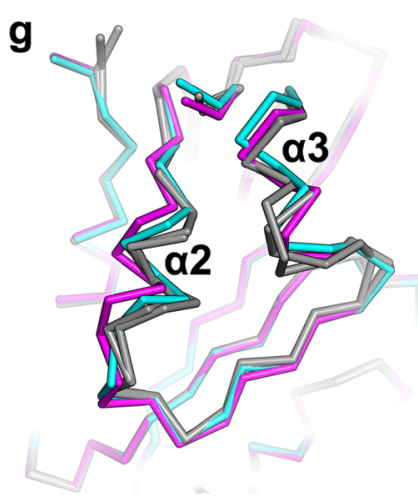


Extended Data Fig. 5 | Comparison of EY6A, ACE2, CR3022 and their epitopes on the RBDs of SARS-CoV-1 and SARS-CoV-2. a, Sequence alignment of RBDs of SARS-CoV-2 and SARS-CoV-1. Residue numbers are those of SARS-CoV-2 RBD, conserved amino acids have a red background, secondary structures are labeled on the top of the sequence, and the glycosylation site is marked with a blue hexagon. Residues involved in receptor binding are marked with magenta disks. Residues shielded by Fab binding are marked with disks: blue indicate heavy chain, cyan light chain and green both chains. b-e, Open book views showing buried solvent accessible surface due to RBD-EY6A complex formation (b, c) and RBD-CR3022 complex formation (d, e). The color scheme is as in (a). f, Superposition of EY6A and CR3022 bound RBDs showing the structural differences at the epitope region. EY6A-bound RBD is shown in magenta and CR3022 bound RBD in cyan. $\mathbf{g}$, Structural differences between EY6A and CR3022 bound RBDs and ACE2 bound RBDs (gray; PDB 6MOJ and 6LZG). h, Comparison of binding modes between EY6A (red, heavy chain; blue, light chain) and VHH72 (gray) in the crystal structure of SARS-CoV-1 RBD-VHH72 complex (PDB 6WAQ). 


\section{ARTICLES}

a

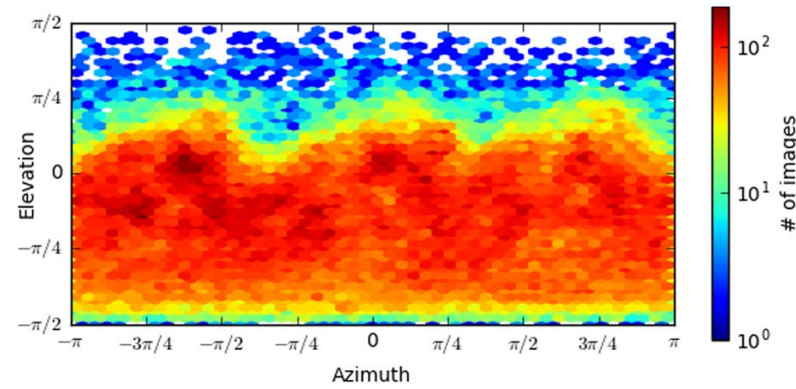

C
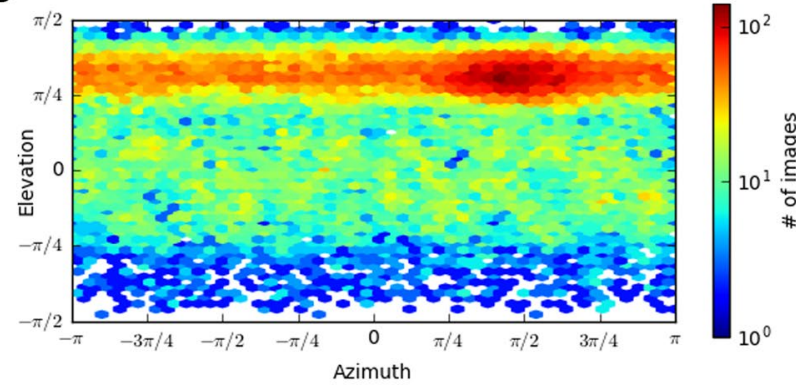

b

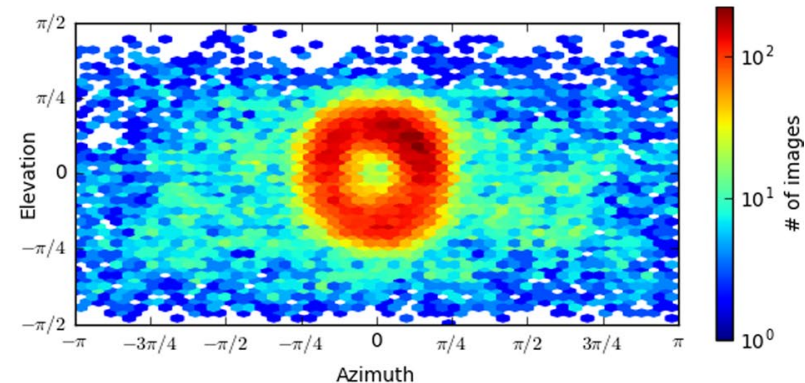

d

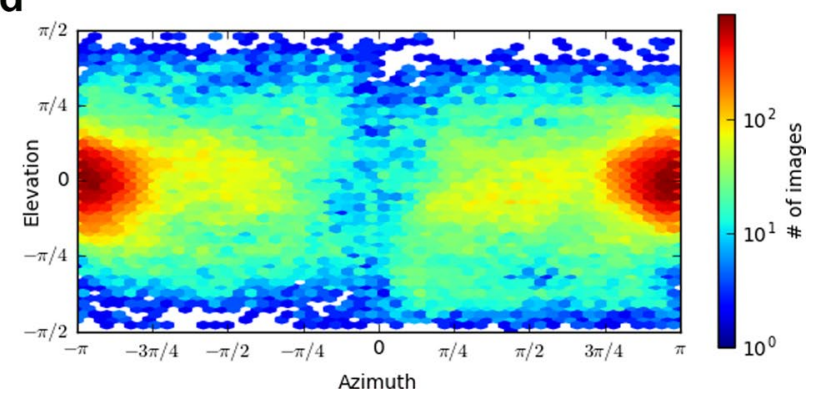

Extended Data Fig. 6 | Cryo-EM Structure view direction distribution plots. a, EY6A-bound intact Spike; b, EY6A-RBD ring with C1 and c, with C3 symmetry imposed. d, EY6A-Spike dimer. Plots were generated within CryoSPARC. See Methods for details. 

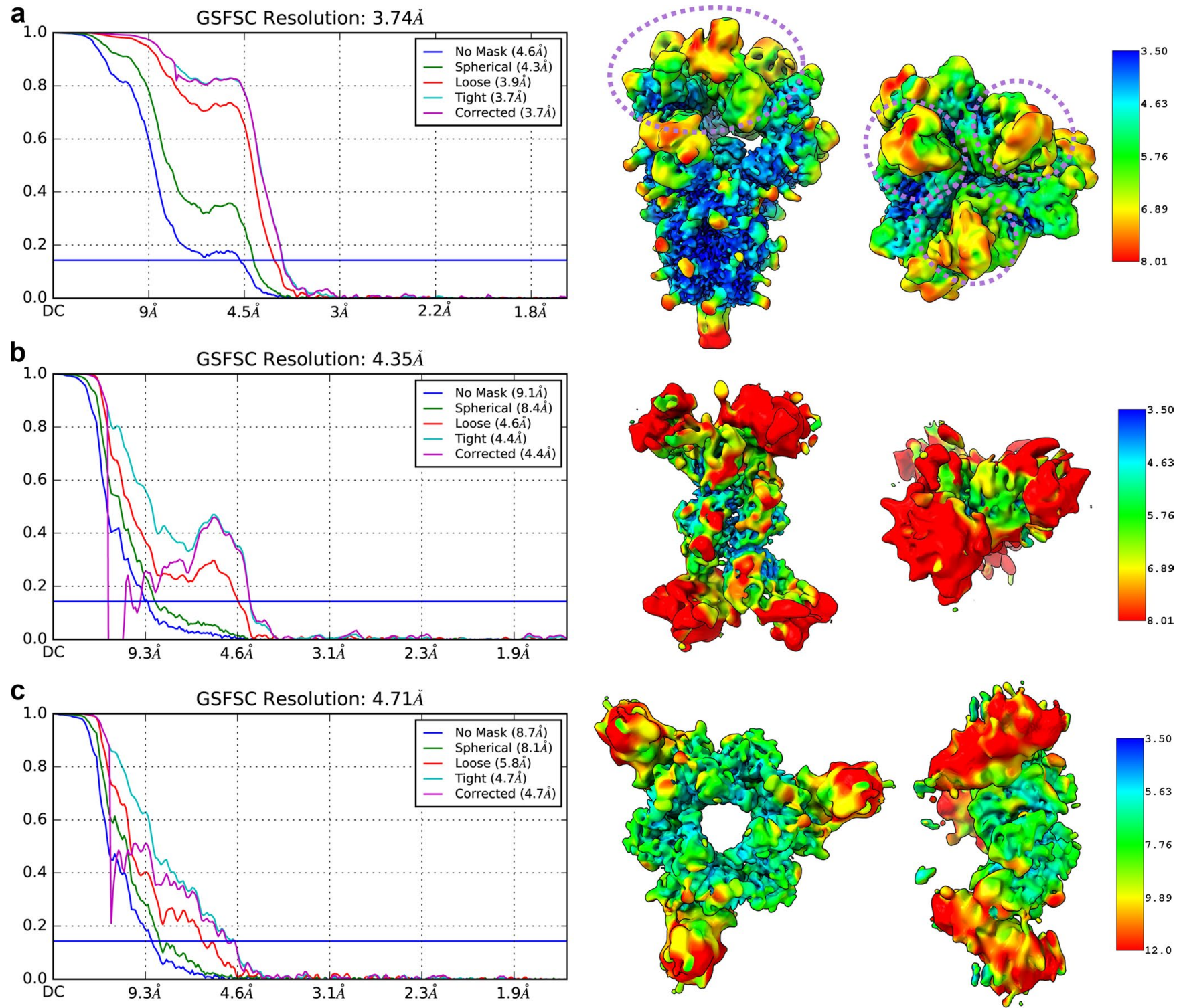

Extended Data Fig. 7 | FSC curves and resolution maps for cryo-EM reconstructions. a, EY6A-bound intact Spike (the purple dotted lines mark the RBD positions); $\mathbf{b}$, 'dimeric fragment'; c, 'trimeric fragment'. 
a

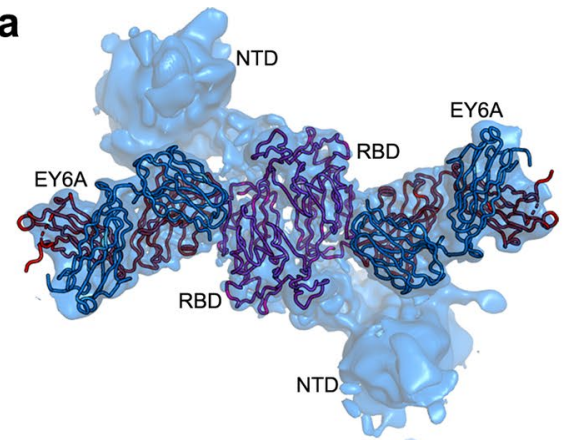

C

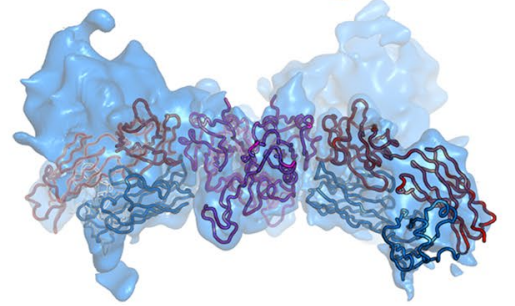

e
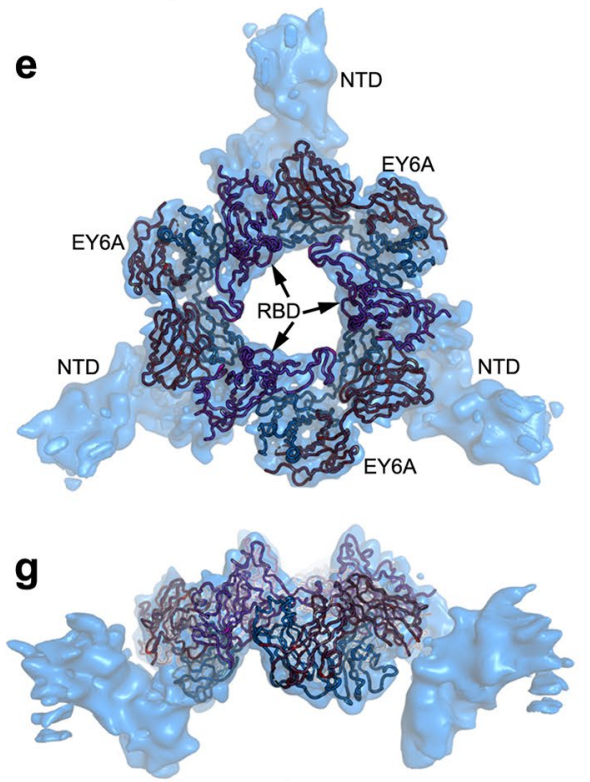

b

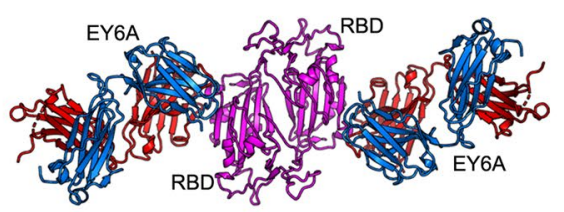

d

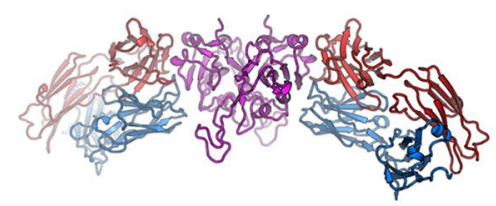

f
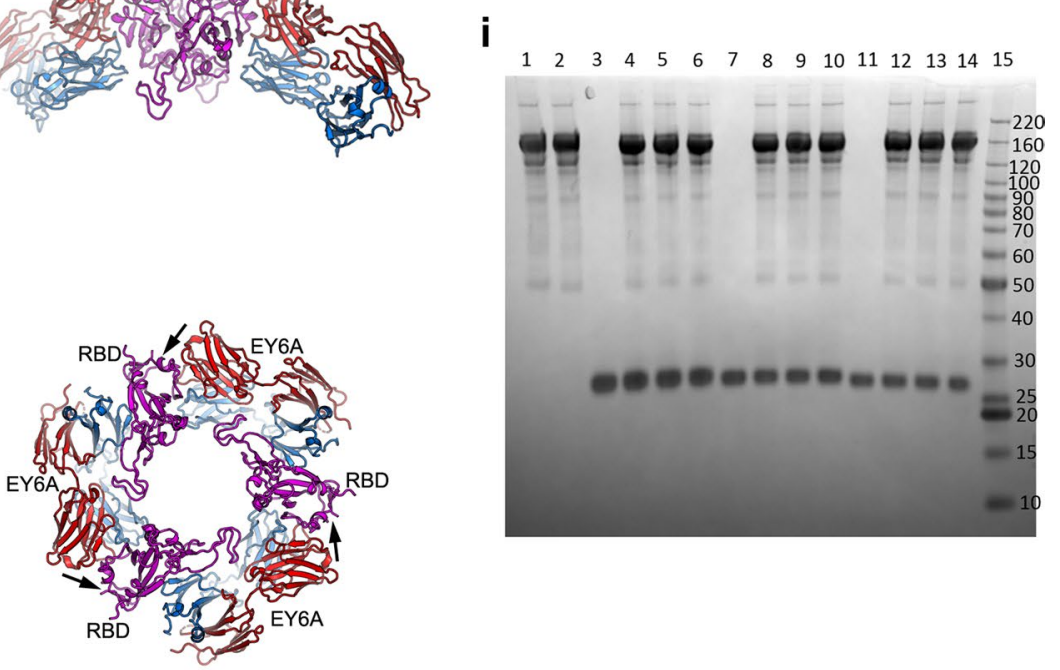

h

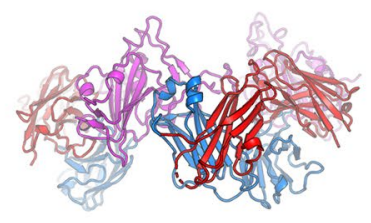

Extended Data Fig. 8 | Analysis of cryo-EM data for $\mathbf{5} \mathbf{h}$ incubation. Final reconstructions derived using an $a b$ initio CryoSPARC reference volume from the $5 \mathrm{~h}$ incubation dataset to show two observed degraded states of Spike-EY6A Fab complex. $\mathbf{a}, \mathbf{b}$, Top view and $\mathbf{c}$, d, side view of the 'dimeric' assembly.

e, $\mathbf{f}$, Top view and $\mathbf{g}$, h side view of the 'trimeric' assembly. EY6A is drawn as a cartoon in blue (light chain) and red (heavy chain), and the RBD in magenta. i, SDS-PAGE analysis of material following $5 \mathrm{~h}$ incubation, as for cryo-EM. The gel is a 4-12\% gradient SDS-PAGE gel (under reducing conditions). Lanes 1 \& 2 are Spike alone. Lane 3 is CR3022 Fab alone, lanes 4-6 are Spike incubated with CR3022 Fab. Lane 7 is EY6A Fab alone, lanes 8-10 are Spike incubated with EY6A Fab. Lanes 11-14 are a non-RBD binding Fab alone and incubated with Spike. Lane 15 is molecular weight markers (kDa). 


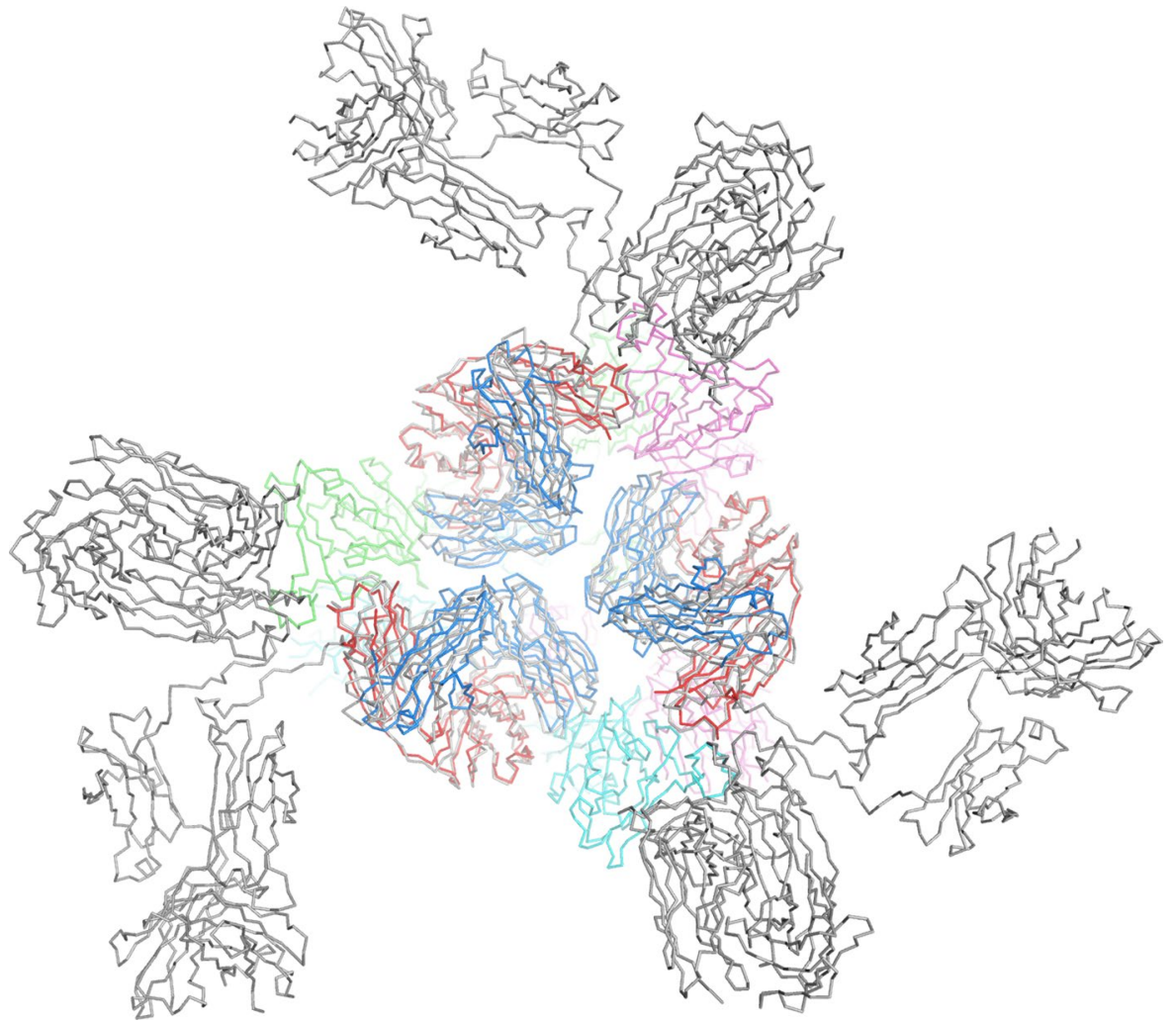

Extended Data Fig. 9 | Intact EY6A antibodies can pack similarly to EY6A Fabs at the pre-fusion Spike head. Representative antibody structures (PDB 1IGT) have been superposed on the EY6A Fab without significant clashes. The Spike chains are colored green, cyan and magenta with EY6A heavy and light chains shown in red and blue respectively and the remainder of the antibodies in gray. 


\section{Reporting Summary}

Nature Research wishes to improve the reproducibility of the work that we publish. This form provides structure for consistency and transparency in reporting. For further information on Nature Research policies, see our Editorial Policies and the Editorial Policy Checklist.

\section{Statistics}

For all statistical analyses, confirm that the following items are present in the figure legend, table legend, main text, or Methods section.

$\mathrm{n} / \mathrm{a}$ Confirmed

\ The exact sample size $(n)$ for each experimental group/condition, given as a discrete number and unit of measurement

$\square$ A statement on whether measurements were taken from distinct samples or whether the same sample was measured repeatedly

The statistical test(s) used AND whether they are one- or two-sided

Only common tests should be described solely by name; describe more complex techniques in the Methods section.

$\square$ A description of all covariates tested

Х $\square$ A description of any assumptions or corrections, such as tests of normality and adjustment for multiple comparisons

$\square$ A full description of the statistical parameters including central tendency (e.g. means) or other basic estimates (e.g. regression coefficient) AND variation (e.g. standard deviation) or associated estimates of uncertainty (e.g. confidence intervals)

For null hypothesis testing, the test statistic (e.g. $F, t, r$ ) with confidence intervals, effect sizes, degrees of freedom and $P$ value noted Give $P$ values as exact values whenever suitable.

Х $\square$ For Bayesian analysis, information on the choice of priors and Markov chain Monte Carlo settings

Х $\square$ For hierarchical and complex designs, identification of the appropriate level for tests and full reporting of outcomes

Х $\square$ Estimates of effect sizes (e.g. Cohen's $d$, Pearson's $r$ ), indicating how they were calculated

our web collection on statistics for biologists contains articles on many of the points above.

\section{Software and code}

Policy information about availability of computer code

Data collection GDA, SerialEM

Data analysis Xia2-dials,Phaser,REFMAC5,COOT,PHENIX,SHP,PISA,PyMOL, Relion 3.1, CryoSPARC v.2.14.1,Gctf-v1.06, Biacore T200 Evaluation Software 3.1, GraphPadPrism8. Graphs were also presented by Microsoft Excel for Mac 2011 version 14.7.7, Microsoft PowerPoint for Mac 2011 version 14.7.7, and GraphPad Prism version 5 softwares. To determine the individual gene segments employed by VDJ and VJ rearrangements and the number of nucleotide mutations and amino acid replacements, the variable domain sequences were aligned with germline gene segments using the international ImMunoGeneTics (IMGT) alignment tool (http://www.imgt.org/IMGT_vquest/vquest).

For manuscripts utilizing custom algorithms or software that are central to the research but not yet described in published literature, software must be made available to editors and

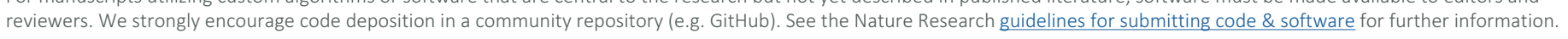

\section{Data}

Policy information about availability of data

All manuscripts must include a data availability statement. This statement should provide the following information, where applicable:

- Accession codes, unique identifiers, or web links for publicly available datasets

- A list of figures that have associated raw data

- A description of any restrictions on data availability

All the data supporting the findings of this study are available within the paper and extended data files. Requests for antibody material should be addressed to Kuan-Ying A. Huang. The coordinates and structure factors for the SARS-CoV-2 RBD-EY6A and SARS-CoV-2 RBD-EY6A-Nb have been deposited in the wwPDB with accession codes 6ZER and 6ZCZ respectively. EM maps and structure models are deposited in EMDB and PDB with accession codes EMD-11174 and 6ZDH (Spike- 
EY6A), EMD-11184 and 6ZFO, and EMD-1173 and 6ZDG (the dimeric and trimeric complexes of the largely structurally disordered Spike ectodomain with bound EY6A Fab, respectively) for immediate release.

\section{Field-specific reporting}

Please select the one below that is the best fit for your research. If you are not sure, read the appropriate sections before making your selection. \ Life sciences $\quad \square$ Behavioural \& social sciences $\quad \square$ Ecological, evolutionary \& environmental sciences

For a reference copy of the document with all sections, see nature.com/documents/nr-reporting-summary-flat.pdf

\section{Life sciences study design}

All studies must disclose on these points even when the disclosure is negative.

Sample size We have isolated antibodies from one SARS-CoV-2-infected donor we enrolled and all the samples we obtained. 360 degrees of data was collected from each of the binary and ternary complex crystals, for the former the redundancy was 19.8 and for the latter 9.4. For the SpikeEY6A complex 144,680 particles were used, for the C3 trimer, 41,372 particles were used, for the C1 dimer, 119,343 particles were used.

Data exclusions For Cryo-EM poor CTF fits were discarded.

Replication All attempts at replication were successful. Data were collected from more than one batch of material and more than one crystal/grid.

Randomization For the cryo-EM, particles were picked by unbiased blob-picking in cryoSPARC.

Blinding For the cryo-EM processing, Relion/cryoSPARC perform unbiased 2D and 3D classification

\section{Reporting for specific materials, systems and methods}

We require information from authors about some types of materials, experimental systems and methods used in many studies. Here, indicate whether each material, system or method listed is relevant to your study. If you are not sure if a list item applies to your research, read the appropriate section before selecting a response.

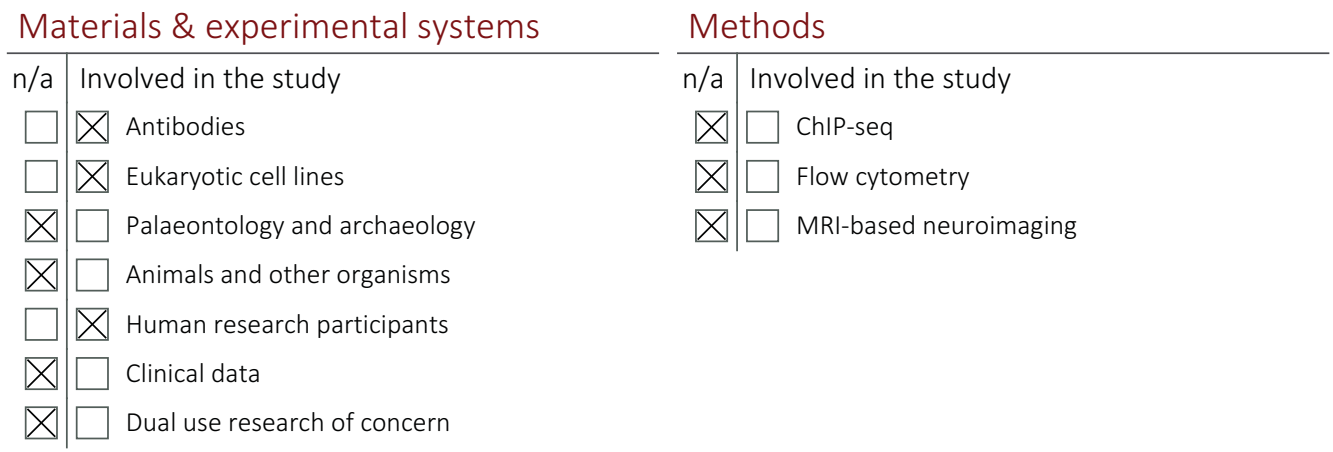

\section{Antibodies}

Antibodies used

For identification of human plasmablasts for production of monoclonal antibodies, Pacific Blue Mouse anti-Human CD3 (clone UCHT1, catalog number 558117, BD)(5 $\mu \mathrm{g} / \mathrm{ml})$, FITC Mouse anti-Human CD19 (clone HIB19, catalog number 555412, BD)(1:10 dilution in a $100 \mu$ l experimental sample), PE-Cy7 Mouse anti-Human CD27 (clone M-T271, catalog number 560609, BD)(1:20 dilution in a $100 \mu$ l experimental sample), APC-H7 Mouse anti-Human CD20 (clone L27, catalog number 641396, BD)(5 $\mu \mathrm{g} / \mathrm{ml})$, PE-Cy5 Mouse anti-Human CD38 (clone HIT2, catalog number 555461, BD)(1:10 dilution in a $100 \mu$ l experimental sample) were used.

For characterization of human antibodies, the following secondary antibodies were used:

Rabbit anti human IgG conjugated with horseradish peroxidase (polyclonal, catalog number 609-4312, Rockland) (1:5000 dilution) Goat anti human IgG labeled with FITC (polyclonal, catalog number H10301, Life Technologies) (1:160 dilution)

More detailed information are available upon request.

Validation

All antibodies used were tested with appropriate negative and positive control samples. The information of all antibodies has been provided above and in the manuscript. 
Policy information about cell lines

Cell line source(s)

Vero E6 (ECACC 85020206, PHE, UK), ExpiCHO (Life Technologies), Expi293TM ( Thermo Fisher), 293T (Sigma-Aldrich) HEK 2935 GnTI (ATCC CRL-3022)

Authentication

All cell lines were frequently checked for cellular morphologies, growth rates and functions, but none of cell lines were authenticated.

Mycoplasma contamination

All cell lines were tested for mycoplasma and found to be mycoplasma-negative (MycoAlert Assay, Lonza and A2H 85011441, Sigma-Aldrich).

Commonly misidentified lines

(See ICLAC register)

No commonly misidentified cell lines were used

\section{Human research participants}

Policy information about studies involving human research participants

Population characteristics

Four human donors, one adult who was diagnosed with COVID-19, were prospectively enrolled in Taoyuan General Hospital, Ministry of Health and Welfare, Taiwan. Naturally occurring acute SARS-CoV-2 infection was diagnosed by positive real-time reverse transcriptase polymerase chain reaction results of respiratory samples according to the guidelines of the Taiwan Centers for Disease Control.

Recruitment

The donor was prospectively enrolled and provided signed informed consent.

Ethics oversight

The study protocol and informed consent were approved by the ethics committee at the Chang Gung Medical Foundation and the Taoyuan General Hospital, Ministry of Health and Welfare, Taiwan. The study and all associated methods were carried out in accordance with the approved protocol and the Declaration of Helsinki and Good Clinical Practice guidelines.

Note that full information on the approval of the study protocol must also be provided in the manuscript. 